\title{
Design of a pressure modulator using fast-acting bistable valves
}

\begin{tabular}{|c|c|}
\hline Journal: & Part C: Journal of Mechanical Engineering Science \\
\hline Manuscript ID & JMES-18-1724 \\
\hline Manuscript Type: & Original article \\
\hline $\begin{array}{r}\text { Date Submitted by the } \\
\text { Author: }\end{array}$ & 01-Dec-2018 \\
\hline Complete List of Authors: & $\begin{array}{l}\text { Henderson, Leon; Chalmers University of Technology, Department of } \\
\text { Mechanics and Maritime Sciences } \\
\text { Cebon, David; University of Cambridge, Engineering }\end{array}$ \\
\hline Keywords: & $\begin{array}{l}\text { Mechanical Modelling, Pneumatics, Actuators, Vehicle Engineering, } \\
\text { Magnetic Device }\end{array}$ \\
\hline Abstract: & $\begin{array}{l}\text { Fast-acting pneumatic valves, combined with a slip-control braking } \\
\text { algorithm, have recently been used to improve the straight-line braking } \\
\text { performance of an experimental heavy goods vehicle (HGV), on low } \\
\text { friction roads, by } 16 \% \text {. This paper describes how the fast-acting valves, } \\
\text { which were central to the aforementioned research, were designed for } \\
\text { use on a commercial vehicle. Design equations, as well as a generalised } \\
\text { design method, are first presented for the fast-acting bistable pneumatic } \\
\text { valve. A pressure observer is developed to predict the brake chamber } \\
\text { pressure in cases where a pressure transducer is mounted upstream. A } \\
\text { simple fault detection algorithm is then introduced, which utilises some } \\
\text { of the calculations made in the pressure observer, and is shown to } \\
\text { correctly identify faults on a real vehicle. Performance comparisons are } \\
\text { made between the new modulator and a conventional HGV electro- } \\
\text { pneumatic brake system. Closed loop frequency response tests show } \\
\text { that the control bandwidth of brake chamber pressure on a HGV can be } \\
\text { increased from } 1.5 \mathrm{~Hz} \text { to } 10 \mathrm{~Hz} \text { using the new hardware. }\end{array}$ \\
\hline
\end{tabular}

\section{SCHOLARONE Manuscripts}




\title{
Design of a pressure modulator using fast-acting bistable valves
}

\author{
${ }^{1, \dagger}$ L. Henderson $\&{ }^{2}$ D.Cebon
}

Submitted to IMechE: Part C, $1^{\text {st }}$ December 2018

${ }^{1}$ Chalmers University of Technology, Department of Mechanics and Maritime Sciences, SE-412 96

Gothenburg, Sweden

${ }^{2}$ University of Cambridge, Department of Engineering, Trumpington St, Cambridge, CB2 1PZ, United Kingdom

${ }^{\dagger}$ Corresponding author: leon.henderson@chalmers.se

\begin{abstract}
Fast-acting pneumatic valves, combined with a slip-control braking algorithm, have recently been used to improve the straight-line braking performance of an experimental heavy goods vehicle (HGV), on low friction roads, by $16 \%$. This paper describes how the fast-acting valves, which were central to the aforementioned research, were designed for use on a commercial vehicle. Design equations, as well as a generalised design method, are first presented for the fast-acting bistable pneumatic valve. A pressure observer is developed to predict the brake chamber pressure in cases where a pressure transducer is mounted upstream. A simple fault detection algorithm is then introduced, which utilises some of the calculations made in the pressure observer, and is shown to correctly identify faults on a real vehicle. Performance comparisons are made between the new modulator and a conventional HGV electro-pneumatic brake system. Closed loop frequency response tests show that the control bandwidth of brake chamber pressure on a HGV can be increased from $1.5 \mathrm{~Hz}$ to $10 \mathrm{~Hz}$ using the new hardware.
\end{abstract}

\section{Keywords}

Solenoid valve, pneumatic, anti-lock braking system, ABS, EBS, slip control, magnetic modelling, brake system design, heavy vehicle 


\section{Introduction}

Heavy goods vehicles (HGVs) exhibit considerably longer emergency stopping distances than automobiles $[1,2]$. They can also experience problems arising from their larger dimensions, high centre of gravity and articulation points. Such issues include: roll-over, jack-knife, trailer-swing and excessive off-tracking [3]. Antilock braking systems (ABS) have been mandatory on HGVs in Europe and the US for some time. These systems prevent wheel lock during braking events on low friction surfaces, reducing the likelihood of jack-knife and trailer swing. In addition to ABS, electronic stability control (ESC) has also recently become common on HGVs, and mandatory on new vehicles in Europe from 2012. ESC and rollstability control (RSC) help improve the lateral performance of the vehicle during cornering events (on low and high adhesion surfaces respectively). This is achieved through automatic braking of particular axles, as well as differential braking between opposite sides of the vehicle.

The performance of ABS, ESC and RSC are limited by the response time and control resolution of the braking system. HGV braking systems are pneumatic, differing from cars which typically use hydraulic brake systems. Their response times are strongly affected by pipe length, pipe diameter and actuator volume. In modern HGV braking systems an 'electronic braking system' (EBS) is employed. These systems still use air as the actuation medium and still employ conventional antilock braking (ABS) pressure control algorithms for emergency stops, but EBS sends demand signals electronically to the pressure modulating valves, reducing signal propagation delays. A typical EBS for an $\mathrm{HGV}$ is shown schematically in Figure 1. As can be seen, driver demand signals are sent from the brake pedal (labelled 6) electronically to local modules (labelled 3, 4 and 5) which regulate the brake pressure at the wheels. Typically, on the front axle of an HGV, normal braking control is achieved using a single electronic proportional relay valve (4). The separate ABS modulator valves shown in Figure 1 (5) only come into effect during ABS or stability control events. Fully pneumatic back-up control circuits are also included to retain functionality if electrical power is lost.

Studies have shown that, by further improving the response time of the pressure control modules at the wheel and implementing a slip-control strategy, the straight-line braking and lateral stability of an articulated HGV can be improved when compared to a modern HGV EBS $[4,5]$. Miller et al. [4] presented straight-line braking tests obtained on a hardware-in- 
the-loop (HiL) test rig, using prototype fast-acting, high flow-rate, bistable pneumatic valves. The improvements seen using the prototype system in this study were significant; with stopping distance and air consumption reduced by $23 \%$ and $25 \%$ respectively compared to a modern semitrailer EBS. Although these improvements were promising, the prototype valves exhibited several undesirable attributes; these included: excessive electrical power consumption, inadequate maximum operating pressure and unreliable electronic drive circuitry.

This paper builds upon work presented in [6] and describes how the proof-of-concept fast acting bistable valve prototypes described in $[4,6]$ have been further developed to enable implementation on a HGV. Simplified magnetic equations are presented to predict the magnetic forces within the bistable valve; key features are discussed that allow the bistable valve to operate at the supply pressures and battery voltages common to HGVs; pressure control equations and a brake chamber pressure observer are presented, allowing an aggressive, high-bandwidth pressure control regime to be implemented, even when pressure transducers cannot be mounted on the brake chamber. The pressure observer developed is also shown to provide a simple means of fault detection on a real vehicle.

\section{Fast-acting bistable pneumatic valve design}

A prototype bistable valve, used in a successful vehicle installation [5], is shown in Figure 2a. The valve includes a spring steel flexure which is cantilevered between the two arms of a mild-steel 'C-frame'. Each arm incorporates a stainless steel through-tube with an internal diameter of $8 \mathrm{~mm}$, a permanent magnet and a pole-piece. The flexure tip can stick to either of the pole-pieces by a magnetic force. A rubber seat is incorporated onto the flexure tip to form an air-tight seal against either of the pole-piece rims so that oscillation of the flexure between the pole pieces opens and closes the air-flow path. A copper coil is wound around the flexure; when pulsed with electrical current this releases the valve from its current state 'flicking' the flexure to the opposite pole-piece.

A sectioned view of a single bistable valve in an enclosure is shown in Figure 2b. It can be seen that, although the valve incorporates two symmetrically positioned seats, only one is used to channel air flow. When mounted in an enclosure in this way, the bistable valve is equivalent to a conventional 2-2 (2 port, 2 state) solenoid valve, operating in either an open or 
closed state. For brake pressure modulation, two valve assemblies are required: one intake ('build') valve and one exhaust ('dump') valve.

Figure 3 shows the forces acting on a bistable valve's central flexure. When the coil is inactive (i.e. when $F_{\text {coil }}$ shown in Figure 3 is zero) the flexure is attracted to either of the pole-pieces by a magnetic hold force $\left(F_{m a g}\right)$, hence being referred to as 'bistable'. In order for the flexure to 'stick' against the pole-piece the following condition must hold:

$$
F_{\text {mag }}+\frac{\pi D_{\text {seal }}^{2}\left(P_{u}-P_{d}\right)}{4} \geq F_{\text {flex }}
$$

where $F_{\text {flex }}$ is the bending force required to displace the flexure tip by $\delta_{\text {flex }}$ and $D_{\text {seal }}$ is the diameter of the seal formed between the pole-piece and flexure seat and $P_{u}$ and $P_{d}$ are the absolute upstream and downstream pressures respectively. In the valve design shown, $D_{\text {seal }}$ is approximately equal to the internal diameter of the through-tube. This differs from previous bistable valve designs where a larger O-ring was used to form the seal, increasing the force term in (1), and making switching more difficult. Providing a pulse of electrical current to the coil opposes the magnetic flux in the flexure, therefore reducing $F_{\text {mag }}$, enabling the valve to switch states (via the restoring force $F_{\text {flex }}$ ). In this analysis, the reduction in $F_{\text {mag }}$ achieved by coil activation is considered as an additional opposing force, $F_{\text {coil }}$. To switch the valve's state, $F_{\text {coil }}$ must satisfy the following inequality:

$$
F_{\text {mag }}+\frac{\pi D_{\text {seal }}^{2}\left(P_{u}-P_{d}\right)}{4}<F_{\text {flex }}+F_{\text {coil }}
$$

As indicated in the above equation, the magnitude of $F_{\text {coil }}$ required to switch the valve's state mut exceed the difference between $F_{m a g}$ and $F_{\text {flex }}$. It increases as the pressure differential across the valve rises, and is also affected by the cross sectional area of the seal.

Some interesting differences between this type of bistable valve and a conventional solenoid valve are listed below:

(i) The bistable valve only requires electrical power to change states, whereas a conventional solenoid valve requires a constant electrical current to hold it in an active state.

(ii) The bistable valve's switching speed is governed predominantly by the stiffness and mass of the central flexure, which can be significantly higher and lower, respectively, than a conventional solenoid valve's moving parts. 


\section{Flexure design}

The flexure is the most important component of the bistable valve assembly. Selection of its dimensions and material properties directly influence a range of conflicting performance criteria, including: switching speed, maximum operating pressure and operating life.

Accurately predicting magnetic hold forces in electromagnetic actuator circuits can be difficult due to large nonlinearities and the large number of possible flux paths. Previous researchers have generally used either Finite-Element-Analysis (FEA) magnetics packages (e.g. that used in [7]) or simplified nonlinear magnetic circuit models, which must be solved iteratively $[8,9]$, to calculate the magnetic fluxes around such systems.

A simplified magnetic model was sought by the authors to be used as a design tool for future bistable valve designs, for cases where the overall valve shape would be similar. Figure 4 shows the FEA simulation presented by Miller in [6] for the original bistable valve design. As can be seen, the pole-piece and flexure regions have considerably higher magnetic flux densities than the other parts of the valve. At these flux levels, magnetic saturation becomes significant. As these components approach complete saturation (typically between 1.5-2.2 T for steel alloys), their permeability tends towards that of free space $\left(\mu_{m, 0}\right)$. By contrast, the relative permeability $\left(\mu_{m, n} / \mu_{m, 0}\right)$ of non-saturated low-carbon steel is between 2,400 and 2,800 [10]. The magnetic reluctance of regions such as the c-frame (which is not saturated in Figure 4) can therefore be considered negligible when compared to the large reluctances of the saturated regions. In order to increase the flux that can flow along the flexure, two 'fingers' 
are mounted alongside it, with small air gaps to the wedges on the side of the flexure. These fingers increase the effective magnetic cross section of the flexure, without making it mechanically stiffer [11].

Bearing all of the above observations in mind, a simplified model of the flexure tip was devised to estimate both $F_{m a g}$ and $F_{\text {coil }}$. The thickness of the flexure tip ( $t_{\text {tip }}$, shown in Figure 3 ) is selected to 'choke' the flux in this region to a desired level, therefore controlling the $F_{\text {mag }}$ and $F_{\text {coil }}$ forces directly. Flux paths through the flexure for the two valve operating conditions (coil inactive and coil active) are shown in Figure 5.

For simple magnetic circuits with a single airgap, the magnetic hold force can be estimated by:

$$
F_{m a g}=\frac{\phi^{2}}{2 \mu_{m, 0} A}
$$

where $\phi$ is the magnetic flux that passes through the airgap, $\mu_{m, 0}$ is the magnetic permeability of free space and $A$ is the effective cross sectional area of the air gap [12,13]. This approximation was found to provide reasonable predictions of the attractive force between each of the two pole pieces and the flexure; the difference between these two forces could be used to derive the overall force acting on the flexure tip as follows:

$$
F_{m a g}=\frac{\phi_{1}^{2}}{2 \mu_{m, 0} A_{1}}-\frac{\phi_{3}^{2}}{2 \mu_{m, 0} A_{3}}
$$

where the subscripts shown correspond to the flux paths shown in Figure 5a. Fringing (shown in Figure 5) was taken into account in the definition of the air gap cross sectional areas $\left(A_{l}\right.$ and $A_{3}$ ) by adding the air gap length $l_{n}$ to the outside radius of the pole piece and subtracting it from the inside diameter (as suggested in [14] and used by Miller et al in [6]). For example, $A_{3}$ in Figure 5 is defined as follows:

$$
A_{3}=\frac{\pi\left(D_{\text {pole }}+2 l_{3}\right)^{2}-\pi\left(D_{\text {orifice }}-2 l_{3}\right)^{2}}{4}
$$

where $l_{3}$ is the air gap length shown in Figure 5.

Assuming both $\phi_{1}$ and $\phi_{2}$ are saturated, calculation of the three flux paths shown in Figure 5a can be estimated by the following relationships: 


$$
\begin{aligned}
& \phi_{1}=\min \left(\left[B_{s, p o l e} A_{1} \quad B_{s, \text { flex }} A_{\text {seat }} \quad B_{r, \text { mag }} A_{\text {mag }}\right]\right) \\
& \phi_{2}=\min \left(\left[\begin{array}{ll}
B_{s, \text { flex }} A_{2} & \phi_{1}
\end{array}\right]\right) \\
& \phi_{3}=\phi_{1}-\phi_{2}
\end{aligned}
$$

where $B_{s, f l e x}$ and $B_{s, p o l e}$ correspond to the saturation flux densities (in $\mathrm{Wb} / \mathrm{m}^{2}$ ) of the flexure and pole-piece materials respectively, and the $B_{r, m a g}$ and $A_{m a g}$ terms are the remnant flux density ${ }^{1}$ and cross sectional area of the permanent magnets respectively. $A_{\text {seat }}$ in these equations corresponds to the cross-sectional area of the flexure seat, and is assumed to have the same outside diameter as the pole-piece, but with no central hole.

When the coil is activated, its magnetomotive force opposes the flux in the flexure. As electrical current is increased in the coil the flux is eventually reversed in the flexure region and channelled back towards the other pole-piece. Flux no longer passes through $A_{2}$ (as shown in Figure 5b), and the flux equations become:

$$
\begin{aligned}
& \phi_{1}=\min \left(\left[\begin{array}{lll}
B_{s, \text { pole }} A_{1} & B_{s, \text { flex }} A_{\text {seat }} & B_{r, \text { mag }} A_{\text {mag }}
\end{array}\right]\right) \\
& \phi_{2}=0 \\
& \phi_{3}=\phi_{1}
\end{aligned}
$$

The effective $F_{\text {coil }}$ is calculated by the difference between $F_{m a g}$ calculated using equations 6-8 (the coil inactive case) and equations 9-11 (the coil active case).

Magnetic hold force predictions from the above model were first compared to those obtained from FEA (Figure 4) and Miller's magnetic circuit model for the earlier generation valve. Agreement was found to be good, but comparison to gaussmeter readings indicated the predicted flux densities through the air gaps were approximately $20 \%$ too high. The fluxes used in equation 5 were therefore corrected using a flux leakage coefficient $\left(\beta_{\text {leakage }}\right)$, similar to that used in [9]. Each flux was redefined as follows

$$
\phi_{n, u s e f u l}=\frac{\phi_{n}}{\beta_{\text {leakage }}}
$$

with $\phi_{n}$ coming directly from equations 6-11.

\footnotetext{
${ }^{1}$ The remnant magnetization of a magnetic material is the flux density $(B)$ which remains in the material once an applied field has been removed. For the permanent magnets in this case it represents the maximum flux density generated by the magnet in an effective 'short-circuit' case.
} 
A comparison of the magnetic hold force predicted by the above model (with $\beta_{\text {leakage }}=1.3$ ) to experimental results for three pole-piece/flexure combinations plotted as a function of polepiece diameter and flexure tip thickness is shown Figure 6. As can be seen, the model fits the experimental data reasonably well. The derivation of $F_{\text {coil }}$ (the difference between the coil active and coil in-active cases) is also shown schematically in the figure, where the ' $0 \mathrm{~mm}$ Flexure' data represents a case where no flux passes through the flexure to the yokes $\left(A_{2}\right.$ in Figure 5). As can be seen, increasing the flexure tip thickness from $1.7 \mathrm{~mm}$ to $3.4 \mathrm{~mm}$ approximately doubles $F_{\text {coil }}$.

Figure 6 also shows the effect of pole-piece diameter on magnetic hold force. At a fixed flexure tip thickness, $D_{\text {pole }}$ can be selected to maximise the magnetic hold force. This strategy is often referred to as pole-piece focusing. Three distinct regions can be seen in Figure 6 for increasing pole-piece diameter (zones 1-3). In zone 1, flux through the flexure seat is limited by saturation in the pole-piece face; zone 2 corresponds to a saturated flexure seat $\left(A_{\text {seat }}\right.$ in Figure 5) and zone 3 corresponds to full remnant flux being provided by the permanent magnets (i.e. magnetic short circuit). To maximise $F_{m a g}$ for a given flexure thickness, the pole-piece diameter should be selected at the boundary between zones 2 and 3, where the flexure seat is saturated and no further magnetic flux can be provided by the permanent magnets. Beyond this point the achievable $F_{m a g}$ reduces, due to a lower flux density passing through the pole-piece face.

Referring back to equation 1 it can be noted that $F_{\text {flex }}$ (the restoring force acting on the flexure due to bending) must be less than $F_{\text {mag }}$ in order for the flexure to hold to the pole-piece (at zero pressure). The bending properties of the flexure are governed predominantly by the root part of the flexure (i.e. the $t_{\text {root }}$ and $L_{l}$ dimensions shown in Figure 3). In order to simplify the calculation of $F_{\text {flex }}$ for a given flexure displacement at the centre of the pole-piece, the thicker tip region is assumed to have infinite bending stiffness. Standard deflection equations for a beam subject to a transverse force and moment (taking into account the offset at which $F_{\text {mag }}$ acts, $L_{2}$ in Figure 3) can be combined and rearranged to give the following equation for $F_{\text {flex }}$ [15]:

$$
F_{f l e x}=\frac{\delta_{f l e x} E I}{L_{1}\left(\frac{L_{1}^{2}}{3}+L_{1} L_{2}+L_{2}^{2}\right)}
$$


where $E$ is the Young's Modulus of the flexure material, $I$ is the second moment of area of the flexure root region and $\delta_{\text {flex }}$ is the transverse deflection of the flexure tip at the centre of the pole-piece (shown in Figure 3).

The required $\delta_{\text {flex }}$ was selected such that the cross-sectional area available for air flow past the flexure was greater than the orifice diameter of the through-tube $(8 \mathrm{~mm})$, i.e.:

$\pi D_{\text {pole }} \delta_{\text {flex }}>\frac{\pi D_{\text {pole }}^{2}}{4}$

This ensures that the high air flow rates needed for EBS control are achievable. The polepiece angle was set at a fixed value, corresponding to the predicted angle of the flexure tip $\left(\theta_{\text {tip }}\right)$ at the specified flexure deflection $\left(\delta_{\text {flex }}\right)$ and was calculated using equation 15 (once again obtained from standard beam deflection equations for an applied force and moment) [16].

$\theta_{t i p}=\frac{F_{f l e x} L_{1}^{2}+2 F_{f l e x} L_{2} L_{1}}{2 E I}$

Valve switching speed was required to be at least the same as that achieved by Miller's [4] valves (approximately $3-4 \mathrm{~ms}$ ). Switch time for bistable valves is made up of two main components: firstly, the time taken for electrical current to build in the coil, and secondly, the time taken for the flexure to move to the opposite pole-piece. Previous testing had suggested that both of these delays were significant [16]. The overall desired switch time was therefore split equally between the two. The time taken for the flexure to travel to the other pole-piece is governed by the first natural frequency of vibration of the flexure. The flexure design incorporates significant mass and rotational inertia at its tip. Rayleigh's Principle was used to estimate the value of the first natural frequency $\left(\omega_{l}\right)$ of the thick tip flexure design. This method calculates the approximate natural frequency $\left(\omega_{n}\right)$ for a specified mode shape as [17]:

$\omega_{n}^{2} \cong \frac{V_{\max }}{\widetilde{T}_{\max }}$

where $V_{\max }$ is the maximum potential energy of the vibration mode, and $\widetilde{T}_{\max }$ is a maximum kinetic energy term with the time derivatives removed [17]. Substituting in the relevant functions for a point mass and inertia (the thick flexure tip) positioned at the end of a uniform beam (the flexure root), equation 16 becomes: 


$$
\omega_{n}{ }^{2} \cong \frac{\int_{0}^{L_{1}} \frac{1}{2} E I\left(\frac{d^{2} u}{d x_{\text {flex }}^{2}}\right)^{2} d x}{\int_{0}^{L_{1}} \frac{1}{2} \rho_{\text {root }} A_{\text {root }} u^{2} d x_{\text {flex }}+\frac{1}{2} m_{\text {tip }} u\left(L_{1}\right)^{2}+\frac{1}{2} J_{\text {tip }} \theta\left(L_{1}\right)^{2}}
$$

where $u\left(x_{f l e x}\right)$ is the mode shape, $x_{f l e x}$ is position along the longitudinal axis of the flexure root (shown in Figure 3), $\theta\left(x_{\text {flex }}\right)$ is the flexure angle at position $x_{f l e x}$ (i.e. $\left.d u / d x_{f l e x}\right), m_{\text {tip }}$ is the mass of the flexure tip and $J_{t i p}$ is the moment of inertia of the tip region taken about the bending axis at the end of the thin part of the flexure $\left(x_{f l e x}=L_{1}\right)$.

In order to obtain a reasonable estimate of $\omega_{1}$ a simple cubic mode shape was assumed. This provided zero displacement and gradient at $x=0$, and maximum displacement, gradient and curvature at $x_{f l e x}=L_{1}$.

Substituting this into equation 17 gives the following estimate of $\omega_{1}$ :

$$
\omega_{1}^{2} \cong \frac{12 E I}{\frac{\rho A_{\text {root }} L_{1}^{4}}{7}+m_{t i p} L_{1}^{3}+9 J_{t i p} L_{1}}
$$

Comparing the natural frequency obtained from equation 18 to that obtained by an FEA analysis (Abaqus) for a model of the thick tip flexure indicated that $\omega_{1}$ estimates using the above approximation were around 10\% higher than the FEA solution. This error was considered to be sufficiently small, for equation 18 was to be used as a design tool to estimate how flexure design changes would influence the valve's switching time, avoiding the need to build detailed FEA model for each design change.

Assuming that the flexure travels from one seat to the other with a motion approximating half a sine wave, the mechanical switching time will be $\pi / \omega_{1}$. The total switching time $\left(\Delta t_{\text {switch }}\right)$ can therefore be estimated as:

$$
\Delta t_{\text {switch }}=\pi / \omega_{1}+\tau_{\text {coil }}
$$

where $\tau_{\text {coil }}$ is the rise-time of the electrical current in the coil.

In order to estimate the fatigue life of the flexure (due to cyclic bending of the flexure root), bending stress $\left(\sigma_{\text {root }}\right)$ at the base of the flexure was calculated using Equation 24. 


$$
\sigma_{\text {root }}=\frac{F_{\text {flex }}\left(L_{1}+L_{2}\right) t_{\text {root }}}{2 I} C
$$

with $F_{\text {flex }}$ calculated according to equation 13, C representing the stress concentration factor of the flexure root. As a steel flexure was to be used, setting a maximum allowable $\sigma_{\text {root }}$ to $50 \%$ the ultimate tensile strength of the material ensured a theoretical infinite fatigue life [18].

\section{Pressure switch tests}

The pneumatic supply pressure on the tractor units of HGVs is typically 12 bar, it was therefore necessary for the bistable valve to operate at this pressure before any vehicle implementation could be carried out. Experimental pressure switch tests were carried on various valve and flexure design iterations to ensure that this design goal was met. In this test, the valve was subjected to a fixed pressure difference and the current in the coil was increased until the flexure switched pole pieces. Figure 7 shows the maximum switching pressure achieved relative to magnetomotive force generated by the coil (Amp-turns in the coil windings) for 3 different bistable valve designs. The main features of the different valve generations shown are as follows:

(i) Generation 1: this is the original prototype presented by Miller in [19]. The flexure was flat and made of spring steel. The seal was formed between the pole-piece and flexure using an O-ring on the pole-piece face.

(ii) Generation 2: this flexure design was also presented by Miller in [4]. 'Finger' components were incorporated into the flexure to provide additional flux carrying paths, while not affecting the bending stiffness of the flexure.

(iii) Generation 3: this design once again incorporated additional 'fingers'. These were cantilevered from the base of the flexure, reducing the overall mass of the moving part of the flexure. The flexure tip thickness was increased to carry more flux. The rubber seat was attached to flexure, allowing $D_{\text {seal }}$ to be reduced relative to the previous valve generations.

The improvements achieved by the additional fingers can be seen by comparing the generation 1 and generation 2 results. The additional improvements achieved by the generation 3 valve/flexure design can also be seen. The generation 3 prototype achieved a maximum switching pressure beyond 12 bar (higher pressures could not be tested due to limitations in the compressed air supply). Its results also have a much steeper gradient than 
those obtained for the other two valves. This is due to the reduction of $D_{\text {seal }}$ in the revised rubber seat design which required less force to lift the flexure from the seat at a given pressure.

Predicted $F_{\text {coil }}$ values obtained from equations 6-11 for the generation 1 and generation 3 flexures are superimposed on Figure 7. The prediction for the flat flexure agrees well with the saturation limit that can be seen as a 'knee' in the experimental results. For reference, the magnetomotive force corresponding to a 100 turn (a feasible number of windings that could fit around the flexure in the bistable valves), $2 \Omega$ coil, operating at $24 \mathrm{~V}$ is also shown on Figure 7 at approximately 1200 A-t. HGVs typically use 24V batteries, so any commercial bistable valve system would need to be able to run at this voltage. Clearly the generation 1 and 2 designs are well below the required maximum switch pressure (12 bar) at this point. By contrast the generation 3 valve should be able to switch 12 bar at $24 \mathrm{~V}$.

The preceding sections of this paper have presented various simplified models which can be used to estimate the performance of a bistable valve design. Figure 8 shows a flow chart of the design process, using these equations, to design a bistable valve to meet arbitrary performance metrics. The process is separated into two main steps; first the magnetic requirements are met by choosing appropriate flexure tip dimensions. Then, flexure root dimensions are selected to achieve the desired mechanical performance metrics. As can be seen in Figure 8 the process may require some iteration if the desired valve specifications cannot be met while ensuring an infinite flexure fatigue life.

\section{Closed loop pressure control}

Two separate 2-2 valves are required to modulate air pressure at the brake chamber. In a conventional pneumatic ABS system, this type of valve manifold is referred to as an ABS modulator valve (shown in Figure 1). A CAD model of the bistable modulator assembly (housing 2 valves) is shown in Figure 9; the inlet and outlet valves, along with the pneumatic flow paths into and out of the brake chamber, are clearly shown. The size of this assembly is comparable to a conventional ABS modulator valve, allowing the valves to be mounted close to the brake chamber. 


\section{Pressure controller details}

A flow diagram of the control tasks carried out by a local wheel-slip control module is shown in Figure 10. As can be seen in the figure, the pressure controller receives pressure demands from a wheel slip controller, which is designed to track the maximum available adhesion force between the tyre and road. For more detail regarding the slip control and $F_{x}$ observer blocks shown in Figure 10 please refer to [20]. The pressure control and pressure observer blocks are presented in more detail below.

Flow through each of the bistable valves is controlled via pulse-width-modulation (PWM), where the mark-space ratio $\left(R_{M S}\right)$ of each valve governs how long they spend open during a particular control cycle; for example, when $R_{M S}=0.75$, a valve will be open for $75 \%$ of a single control cycle. $R_{M S}$ is recalculated at the start of each control cycle; a cycle time of $20 \mathrm{~ms}$ was used for the bistable valves in the work described here.

A simple proportional pressure controller was used to define the desired mark-space ratio $\left(R_{M S}\right)$ for the inlet and outlet valves, using the following relationship:

$$
R_{M S}=k_{\text {press }}\left(P_{d e m}-P_{c}\right)
$$

where $P_{c}$ is the brake chamber pressure, $P_{d e m}$ is the demand pressure and $k_{\text {press }}$ is a tuning gain. Here, positive $R_{M S}$ values correspond to an inlet valve demand, and negative $R_{M S}$ corresponding to an outlet valve demand. Two physical changes were made relative to the system used with the earlier bistable valves [16]. Firstly, the main pressure control calculation and the PWM parts of the controller were implemented in a single digital microprocessor, as opposed to the analogue circuitry used in [16]. This change enabled more flexibility in the control algorithms, and reduced the number of electrical components at the wheel station, since the same microprocessor could be used for slip control calculations. The second change was the repositioning of the pressure transducer. In previous HiL testing [4] the pressure transducer was mounted to a second port on the brake chamber. This provided a direct measurement of brake chamber pressure $\left(P_{c}\right)$. However, HGV brake chambers that also incorporate spring-brakes (which are common to most vehicles) only have a single inlet port, making mounting a pressure transducer in this configuration impossible. Including the pressure transducer in the valve enclosure assembly was seen as a more elegant solution, allowing the new valve block assembly with integral pressure transducer to be fitted to a 
range of different brake chambers. The pressure transducer location is marked on the CAD model of the valve enclosure shown in Figure 9.

\section{Preliminary testing}

Preliminary testing of the pressure controller identified a potential problem with the new system layout. Simulations of the original analogue pressure controller by Miller et al [4], had suggested that the minimum proportional gain $\left(k_{\text {press }}\right)$ needed to be close to $2 \times 10^{-5} \mathrm{~Pa}^{-1}$ in order to achieve a control bandwidth above the typical HGV wheel-hop frequency $(12.5 \mathrm{~Hz})$ [4]. A gain of $1.5 \times 10^{-5} \mathrm{~Pa}^{-1}$ was achieved during the HiL testing in [4].

Figure 11 shows the pressures in the valve block enclosure and brake using a $k_{\text {press }}=1.5 \times 10^{-}$ ${ }^{5} \mathrm{~Pa}^{-1}$ with the new bistable valve hardware. Excessive 'chatter' can be seen, as well as a clear difference between $P_{\text {encl }}$ (the valve block enclosure pressure used in the pressure control calculations) and the actual brake chamber pressure $\left(P_{c}\right)$. The large spikes in enclosure pressure seen in Figure 11 are due to the throttling of air through the restrictions between the bistable modulator and the actual brake chamber and the dynamics of the air volume between the modulator and the brake chamber.

A chamber pressure observer was proposed to estimate $P_{c}$ from the measured pressure in the valve enclosure. This is presented in the following section.

\section{Chamber pressure observer}

Detailed models of the air flow through the bistable valves, as well as the brake chamber's volume versus pressure characteristics, were presented in [4]. These provided a good understanding of the brake chamber's response to changes in valve state.

Flow through each of the bistable valve orifices was estimated using one-dimensional fluid flow theory, as described in detail in $[16,20]$. The governing equations are presented below:

$$
\begin{gathered}
\dot{m}_{v}=S_{v} C_{f} A_{v} C_{1} \frac{P_{u}}{\sqrt{T_{\text {cham }}}} \quad \text { if } \frac{P_{d}}{P_{u}} \leq p_{c r} \quad \text { (choked) } \\
\dot{m}_{v}=S_{v} C_{f} A_{v} C_{2} \frac{P_{u}}{\sqrt{T_{\text {cham }}}}\left(\frac{P_{d}}{P_{u}}\right)^{\frac{1}{\gamma}} \sqrt{1-\left(\frac{P_{d}}{P_{u}}\right)^{\frac{\gamma-1}{\gamma}}} \text { if } \frac{P_{d}}{P_{u}}>p_{c r} \text { (non-choked) }
\end{gathered}
$$

where the subscript $v$ corresponds to either the inlet (in) or outlet (out) valve, $S_{v}$ is the valve state (open $=1$, closed $=0$ ), $C_{f}$ is the valve discharge coefficient (determined experimentally 
by Miller in [19]), $A_{v}$ is the cross-sectional area of the valve orifice, $P_{u}$ is the upstream pressure, and $P_{d}$ is the downstream pressure. $C_{1}$ and $C_{2}$ are constants defined by:

$$
C_{1}=\sqrt{\frac{\gamma}{R}\left(\frac{2}{\gamma+1}\right)^{\frac{\gamma+1}{\gamma-1}}} ; C_{2}=\sqrt{\frac{2 \gamma}{R(\gamma-1)}}
$$

$p_{c r}$ is the critical pressure ratio, defining when the air flow through the orifice is choked or non-choked, and is calculated by the equation 26 .

$$
p_{c r}=\left(\frac{2}{\gamma+1}\right)^{\frac{\gamma}{\gamma-1}} \approx 0.5 \text { for air }
$$

The air within the brake chamber volume was modelled as a polytropic gas of the form $P V^{\alpha}=$ const, where $P$ and $V$ are absolute pressure and volume respectively. Brake chamber pressure was therefore described by the following relationship:

$$
\left(\alpha_{\text {in }} \dot{m}_{\text {in }}-\alpha_{\text {out }} \dot{m}_{\text {out }}\right)=\frac{V_{c} \dot{P}_{c}}{R T_{c}}+\frac{\alpha_{c} \dot{V}_{c} P_{c}}{R T_{c}}
$$

where $V_{c}$ is the chamber volume, $R$ is the gas constant for air and $T_{c}$ is the brake chamber temperature.

In order to design a suitable brake chamber pressure observer, the existing brake chamber model (equations 23-27) was extended to include an additional restriction, $C_{c}$, and an additional valve block enclosure volume, $V_{\text {encl }}$ (these are shown schematically in Figure 9). $C_{c}$ and cross-sectional area, $A_{c}$, were obtained via experiment and direct measurement respectively. $\dot{m}_{C}$ (the mass flow rate of air into the brake chamber) was calculated using the flow equations presented in equations 23-26, with $S_{v}$ (valve state) set to 1 . Valve enclosure pressure $\left(P_{\text {encl }}\right)$ was modelled by the following equation:

$$
\left(\alpha_{\text {in }} \dot{m}_{\text {in }}-\alpha_{\text {out }} \dot{m}_{\text {out }}-\alpha_{c} \dot{m}_{c}\right)=\frac{V_{\text {encl }} \dot{P}_{\text {encl }}}{R T_{\text {encl }}}
$$

where $V_{\text {encl }}$ was assumed fixed, and taken directly from the CAD model of the valve enclosure. The $T_{\text {encl }}$ and $\alpha$ values here correspond to the valve block enclosure temperature and the polytropic flow constants respectively.

Equations 23-28 were combined to create a simple non-linear pressure observer, based on an observer design presented by Bigras and Khayati in [21]. The general observer structure was as follows: 
$\left[\begin{array}{c}\dot{P}_{c} \\ \dot{P}_{\text {encl }}\end{array}\right]=\left[\begin{array}{c}\frac{\alpha_{c} R_{c} T_{c}}{V_{c}\left(P_{c}\right)} \dot{m}_{c} \\ \frac{R T_{\text {encl }}}{V_{\text {encl }}}\left(\alpha_{\text {in }} \dot{m}_{\text {in }}-\alpha_{\text {out }} \dot{m}_{\text {out }}-\alpha_{c} \dot{m}_{c}\right)\end{array}\right]+\left[\begin{array}{c}K_{c} \\ K_{\text {encl }}\end{array}\right]\left(P_{\text {encl }}-\hat{P}_{\text {encl }}\right)$

where states with a '^’ represent estimates, and $K_{c}$ and $K_{\text {encl }}$ are observer gains. The $P_{\text {encl }}$ signal shown is the sensor signal obtained from the pressure transducer. Several assumptions and simplifications were made here, so that real-time implementation was feasible. Variable brake chamber volume was only included in the $V_{c}$ term. The $\psi_{c}^{\&}$ term, which was included in [4], was neglected here to reduce computational load. This simplification introduced some inaccuracies in the plant model. The resulting error was, however, considered acceptable as $P_{c} \dot{V}_{c}$ and $V_{c} \dot{P}_{c}$ values calculated for a typical for a chamber fill process (equation 27) indicated that the later term was generally around 2 orders of magnitude larger than the former. All mass flow rate terms were implemented as pre-calculated look-up tables relative to the ratio $P_{d} / P_{u}$ (via equations 23-26).

The observer gains ( $K_{c}$ and $K_{\text {encl }}$ ) were iteratively tuned by post-processing data obtained from HiL experiments. Sample results obtained from the observer are shown in Figure 12a and Figure 12b, for chamber fill and exhaust cycles respectively. Agreement between the actual and observed states is good. Figure 13 shows the closed loop response of the pressure controller to a step pressure demand, using the estimated pressure as the feedback signal for the digital pressure controller. The actual brake chamber pressure data shown is only included as a reference. Comparing Figure 13 to Figure 11, a significant improvement in controller stability can be seen. The chattering seen in the earlier case has been removed completely and the controller now takes approximately $50 \mathrm{~ms}$ to reach steady state (as opposed to $200 \mathrm{~ms}$ for the same pressure controller without an observer).

\section{Fault Detection}

In addition to improving the pressure control response of the bistable valve system, the chamber pressure observer can also be used to detect faults. The $P_{\text {encl }}-\hat{P}_{\text {encl }}$ term in equation 29 provides a measure of how well the measured valve enclosure pressure agrees with the expected value, given the current valve state demands. Monitoring this term can therefore identify failures relating to both bistable valve hardware (e.g. a blocked valve orifice) and the high-current valve drive electronics (e.g. a blown transistor). One advantage of this type of 
fault detection strategy is that the error term is already calculated during the pressure observer calculations, so very little additional computational capacity is required to check for faults.

During the vehicle tests carried out in [5] the fault detection strategy discussed above was implemented in real-time. The $P_{\text {encl }}-\hat{P}_{\text {encl }}$ term was passed through a simple first-order filter with a 0.1 second time constant to reduce the chance of false positive fault detections during pressure transients. An error threshold of 0.5 bar was then used to trigger a 'fault detected' flag which could be used for safety intervention if needed. An example of the fault detector correctly identifying a problem during a constant pressure demand commissioning test is shown in Figure 14. As can be seen, the brake chamber at wheel 1 did not fill correctly in this case (due to a failure in the drive circuit electronics). The failure was correctly identified by the fault detector within 0.1 seconds.

\section{Hardware-in-the-loop testing: bandwidth comparisons}

To control slip in the presence of the wheel-hop vibration mode, a control bandwidth of at least $12.5 \mathrm{~Hz}$ is required [4]. Figure 15 shows sample pressure traces of the generation 2 and generation 3 bistable valves attached to brake chambers, responding to a $10 \mathrm{~Hz}$ pressure demand. The response of a conventional HGV EBS system is also shown for reference. The conventional hardware is not capable of following the sinusoidal brake pressure demand at this frequency. The bistable valves achieve significantly better pressure demand tracking, with the generation 3 showing much better performance than generation 2 .

Miller et al [4] used a sinusoidal chirp signal of $0.75 \mathrm{bar}$ amplitude, centred about 4bar (absolute pressure), to estimate the bandwidth of the bistable valve pressure control loop. The amplitude and offset of the chirp signal were chosen to represent a typical ABS or slip control stop on a low to medium friction road. Identical tests were carried out with the generation 3 system to enable a direct comparison against the previous generation system. The pressure demand chirp signal lasted 30 seconds and spanned a frequency range of $0.5-50 \mathrm{~Hz}$. The same tests were also carried out with a conventional EBS system as a baseline comparison. Figure 16 shows the frequency response (as a Bode plot) of the three systems for frequencies between 1 and $50 \mathrm{~Hz}$. The generation 3 results shown here correspond to the ensemble average responses of 6 prototype valve enclosures. The results for the generation 2 system correspond to the tests carried out by Miller et al in [4]. For linear systems, the bandwidth is defined as the frequency at which a $3 \mathrm{~dB}$ drop in gain occurs. These points are marked on 
Figure 16. By this measure, the generation 3 valve has a bandwidth of $20 \mathrm{~Hz}$ while conventional valves have a bandwidth of $6 \mathrm{~Hz}$. As the system is highly nonlinear, a more realistic measure of bandwidth is the frequency where a $45^{\circ}$ lag in phase occurs (which for linear systems would correspond to the $-3 \mathrm{~dB}$ frequency). These are also marked on Figure 16, with the generation 3 system achieving an apparent control bandwidth of just over $10 \mathrm{~Hz}$. This is significantly higher than both the conventional EBS $(1.5 \mathrm{~Hz})$ and generation 2 bistable valves $(6 \mathrm{~Hz})$.

\section{Conclusions}

(i) Experimental results show that a simplified magnetic model, which assumes magnetic flux through the magnetic circuit is limited by saturation in one or more components, can be used to correctly predict the magnetic hold force and maximum switching force of a bistable pneumatic valve.

(ii) A bistable valve flexure and seat design that incorporates a 'thick tip' and additional magnetic flux channelling components can meet the necessary performance criteria for implementation in a conventional heavy goods vehicle (HGV) pneumatic brake system, (12bar maximum supply pressure, $8 \mathrm{~mm}$ orifice diameter and $24 \mathrm{~V}$ operation). These performance criteria were not met by previous bistable valve designs.

(iii) A pressure observer that includes a non-linear pneumatic model of a brake chamber and its orifices can be used to accurately predict brake chamber pressure with a pressure transducer located at some distance upstream from the brake chamber. This facilitates precise control of pressure in the brake chamber.

(iv) The error term included in a brake chamber pressure observer can be used to identify faults in bistable valve hardware and electronics on a real vehicle while requiring very little additional computational capacity.

(v) Closed loop pressure control tests indicate that a bistable ABS modulator valve connected to a brake chamber with a digital pressure controller can achieve a pressure control bandwidth of $10 \mathrm{~Hz}$, significantly higher than conventional $\mathrm{HGV}$ EBS hardware $(1.5 \mathrm{~Hz})$ and a previous-generation bistable valve system $(6 \mathrm{~Hz})$. 


\section{Figures}

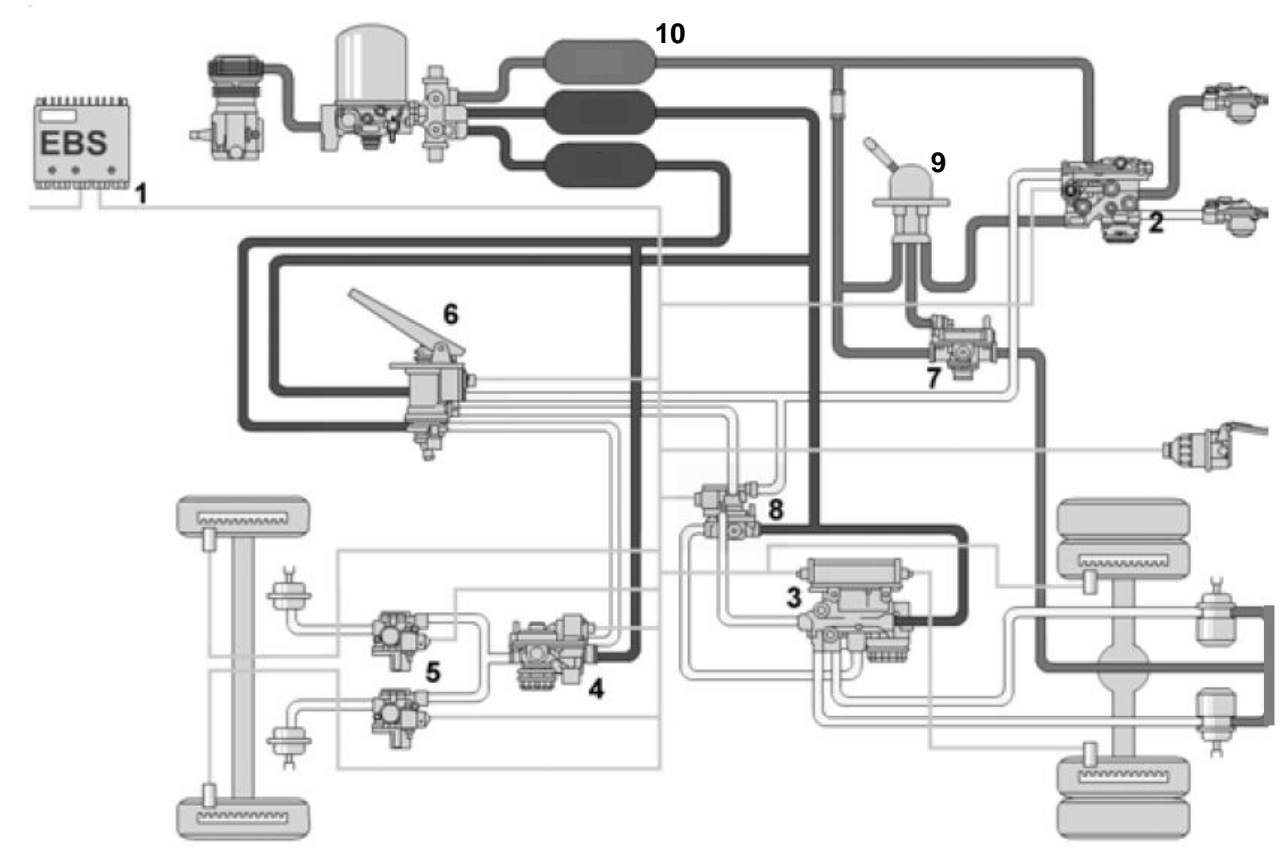

Figure 1: Schematic showing typical heavy goods vehicle (HGV) electronic brake system (EBS) layout [22]; (1) EBS central module, (2) Trailer control valve, (3)Two channel axle modulator, (4) Electronic proportional relay valve, (5) ABS modulator valve, (6) Foot valve, (7) Pneumatic relay valve, (8) Redundancy valve, (9) Park brake control, (10) Compressed air storage tanks. 


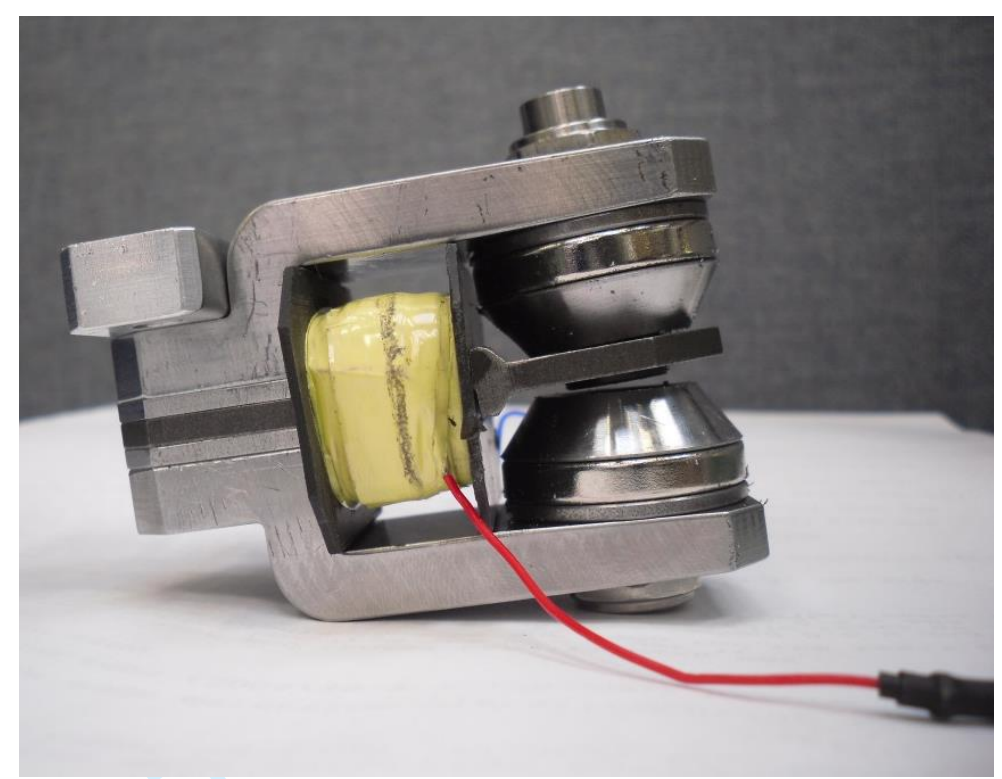

(a)

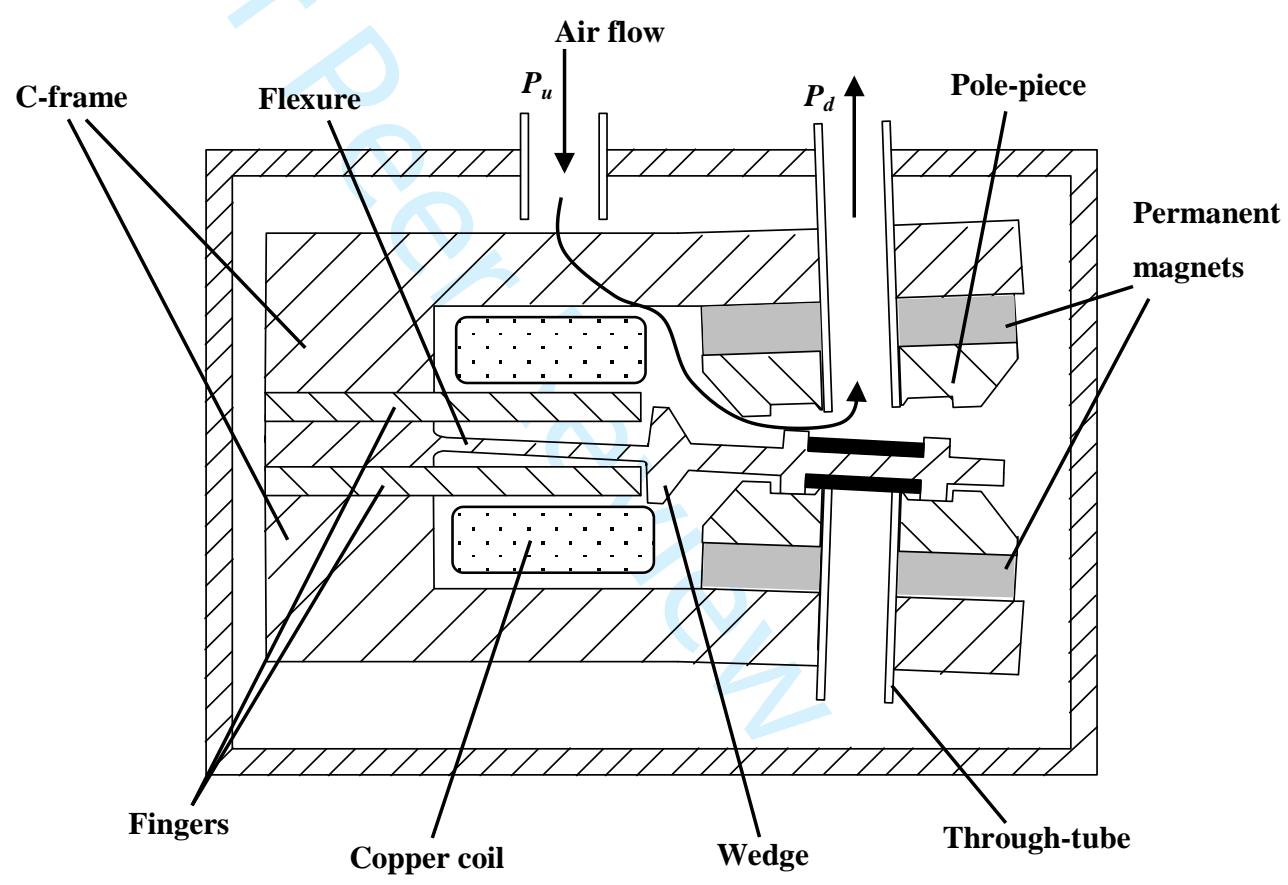

(b)

Figure 2: A bistable pneumatic valve; (a) prototype used in vehicle tests [5], (b) schematic showing single bistable valve in an enclosure 


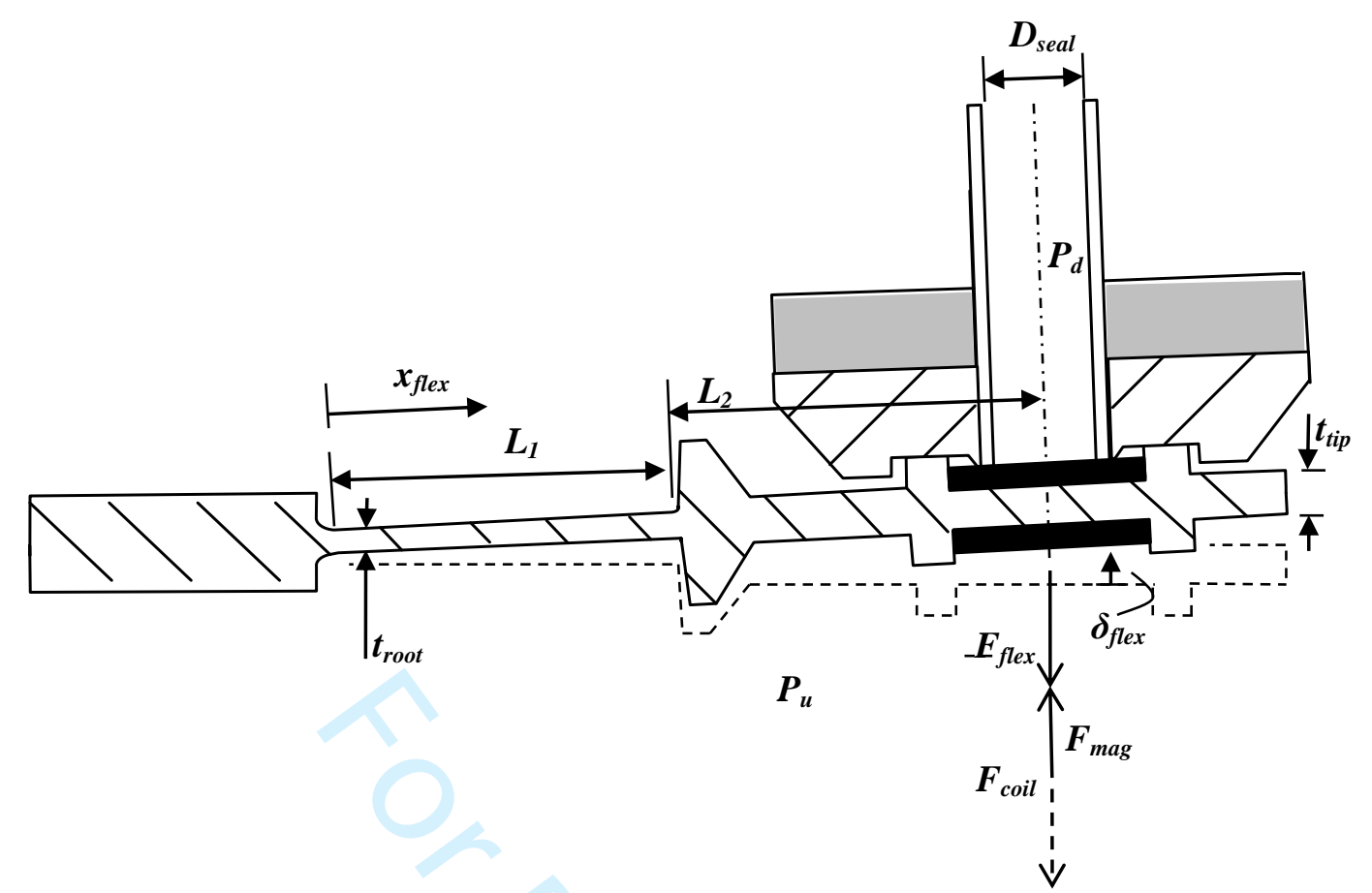

Figure 3: Forces acting on bistable valve flexure 


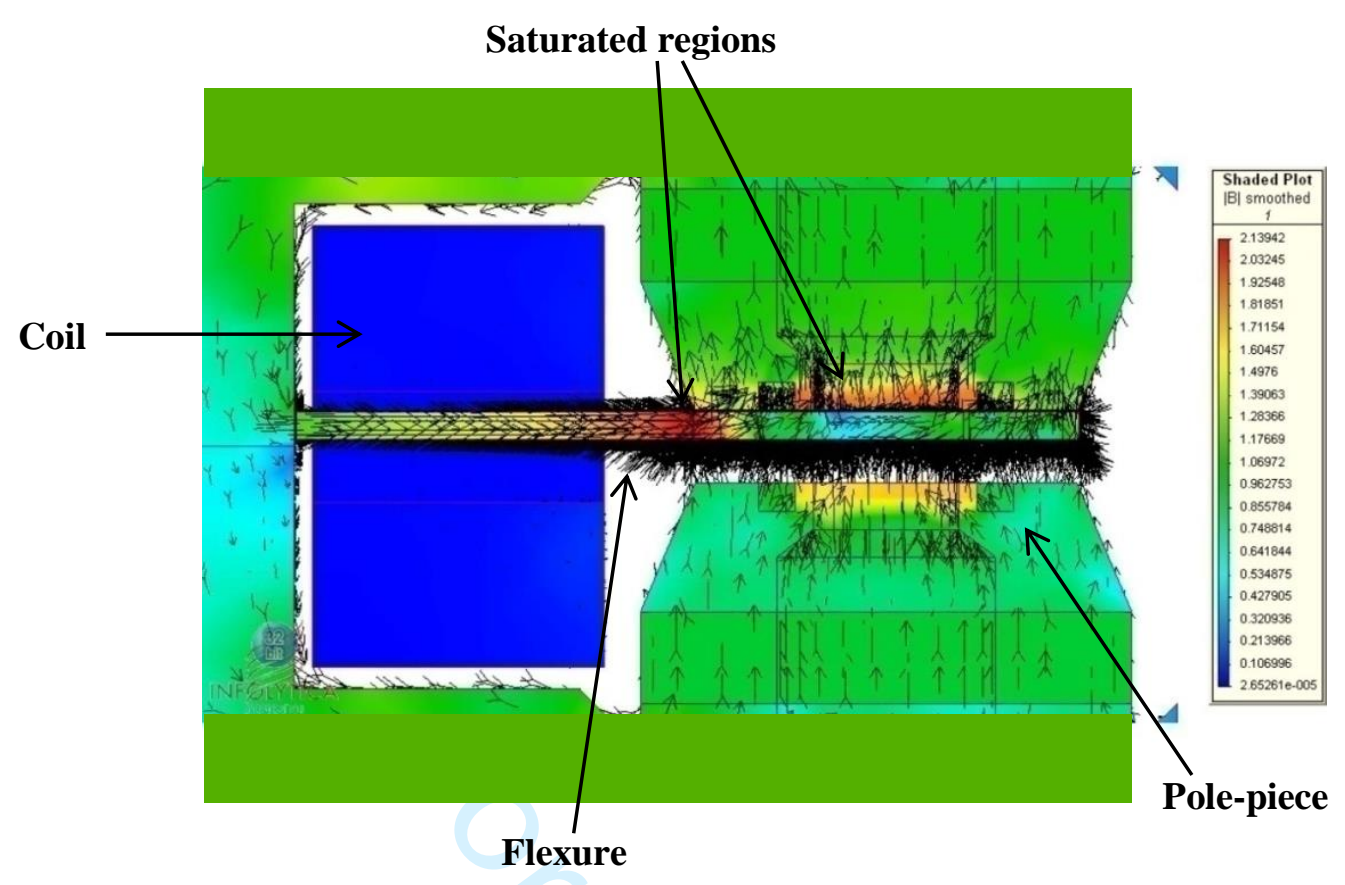

Figure 4: Magnetic flux paths predicted through early bistable valve concept using FEA simulation [6]. Red and blue regions correspond to high and low values of magnetic flux density respectively. 


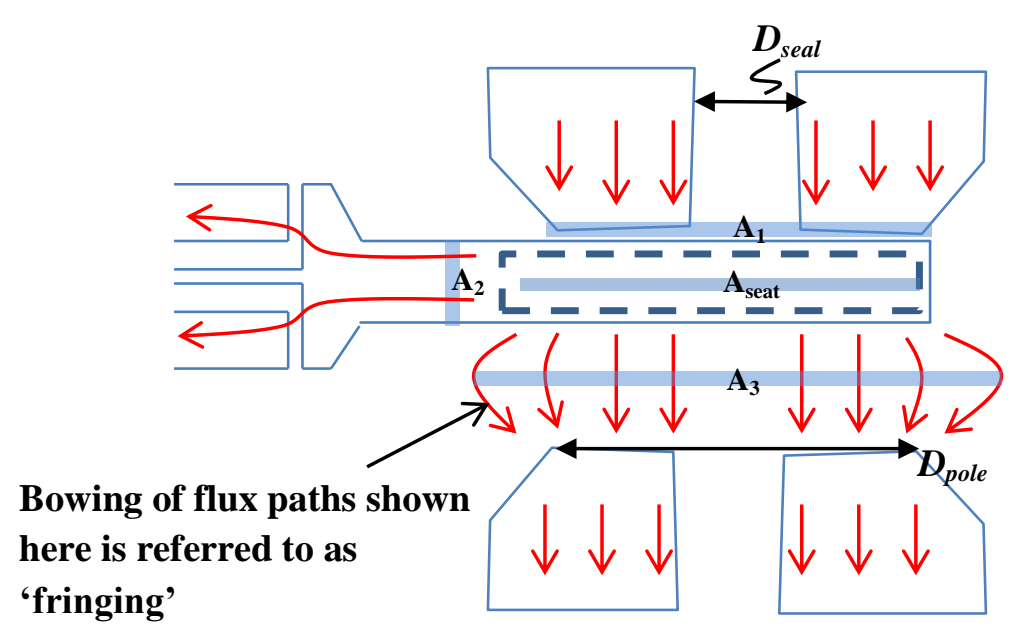

(a)

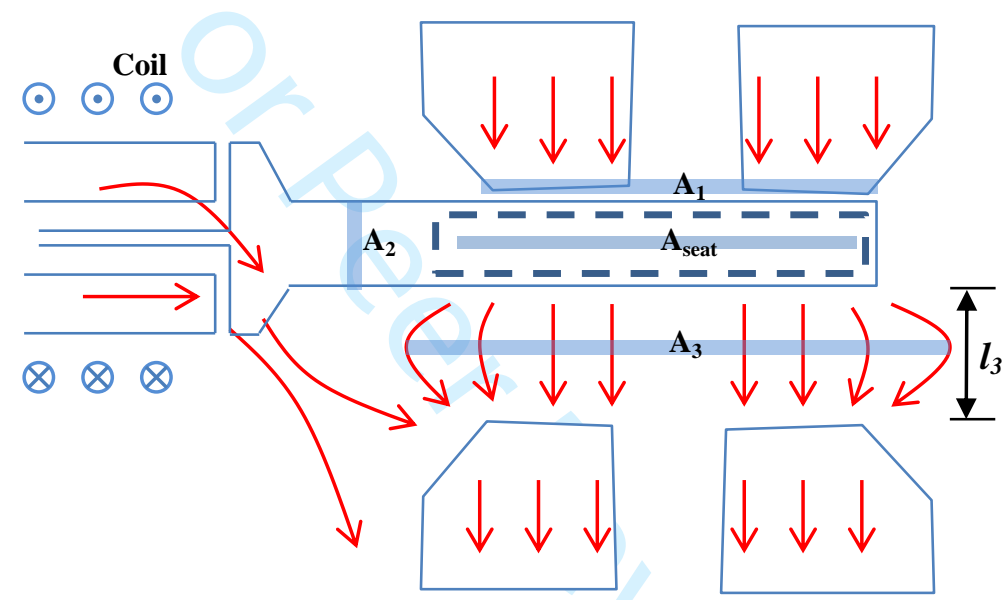

(b)

Figure 5: Flux paths through flexure tip; (a) Coil inactive, flexure held to pole-piece, (b) Coil active, flexure released. Effective areas $A_{1}-A_{3}$ are shown schematically by shaded bars. 


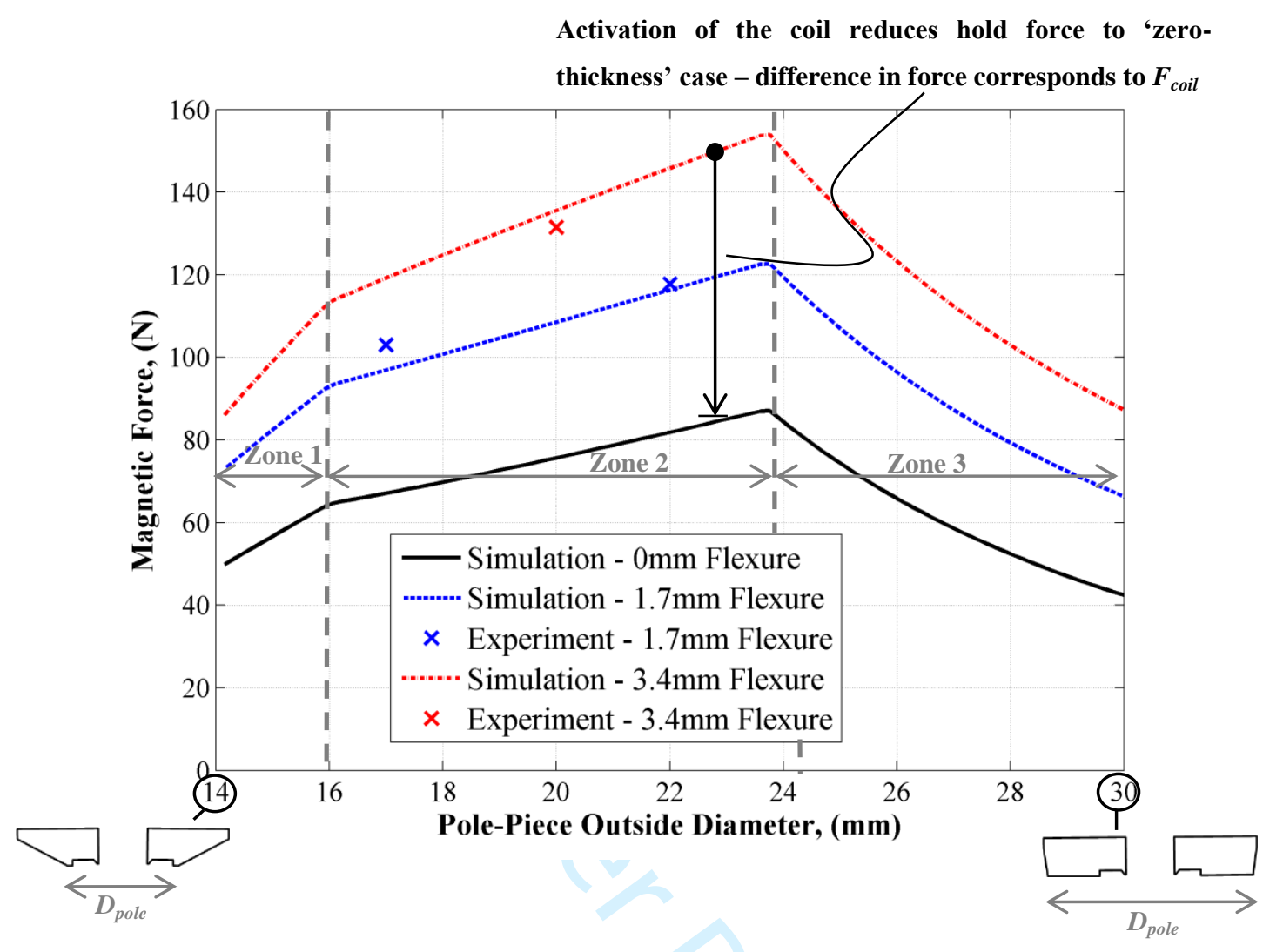

Figure 6: Influence of pole-piece focussing and flexure tip thickness on magnetic hold force $\left(F_{\text {mag }}\right)$ of a bistable valve. Omm flexure corresponds to case where no flux travels through flexure back to the yokes ( $A_{2}$ in Figure 5) 


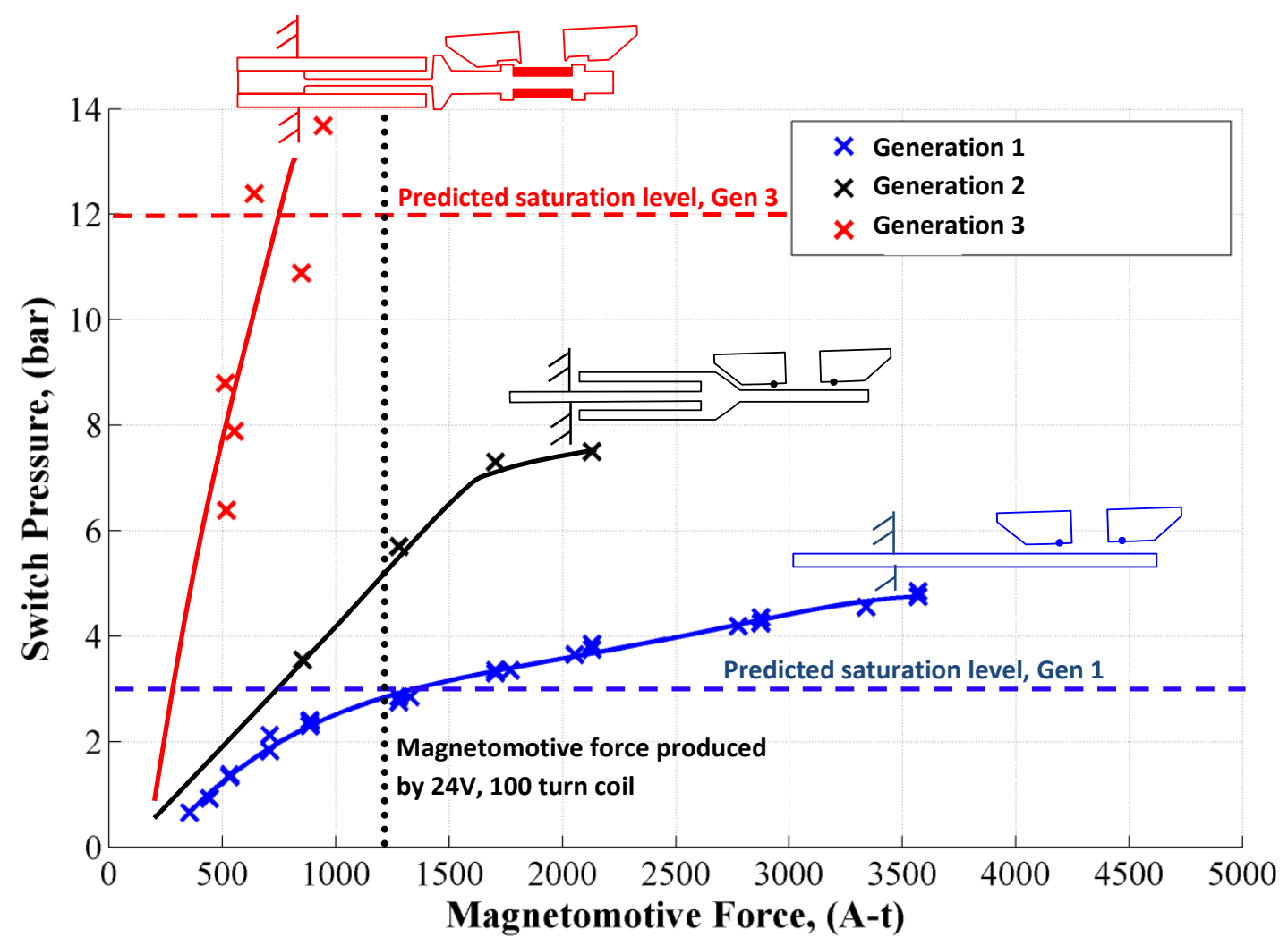

Figure 7: Maximum valve switching pressure, comparing various bistable valve designs 


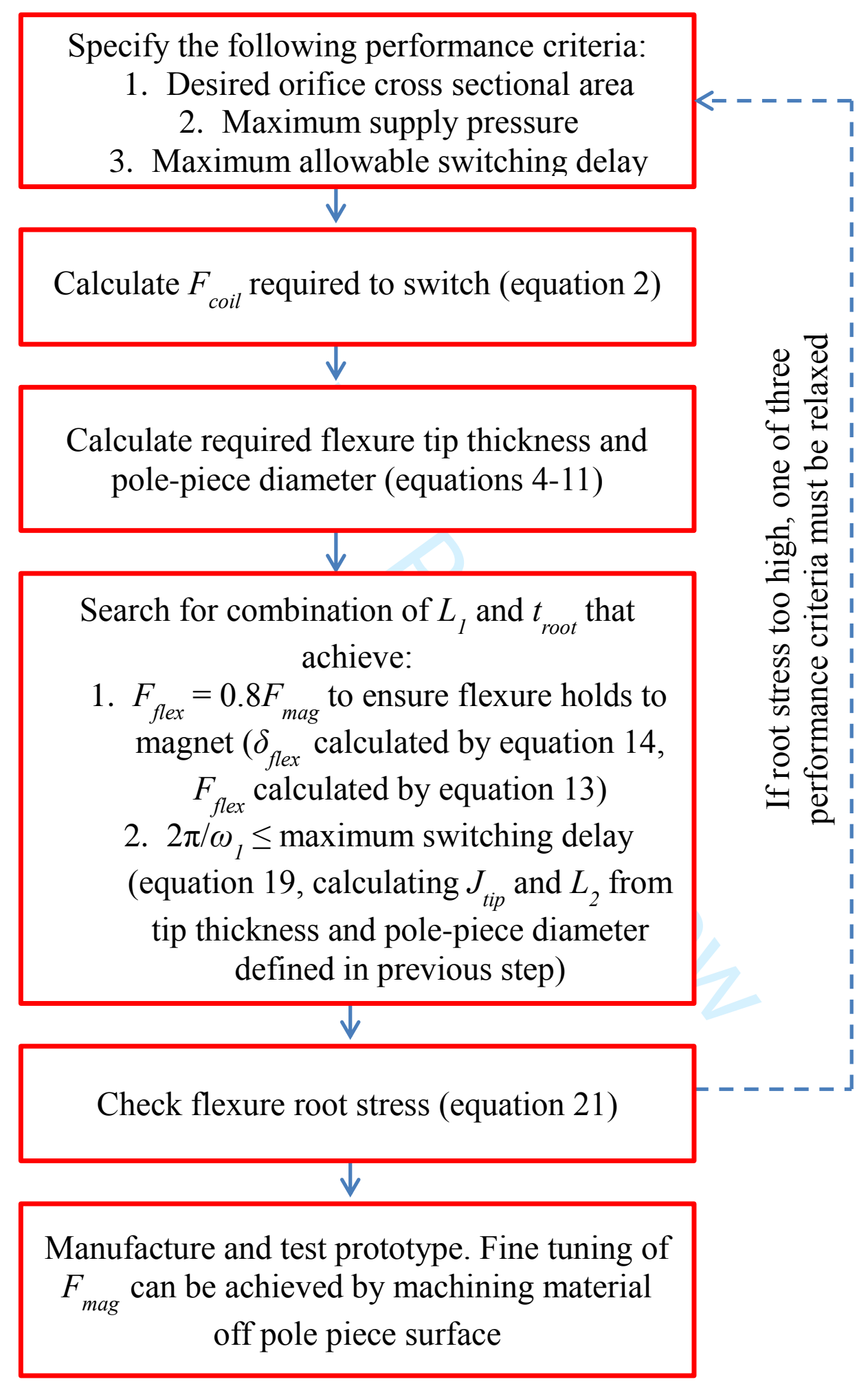

Figure 8: General bistable valve design process 


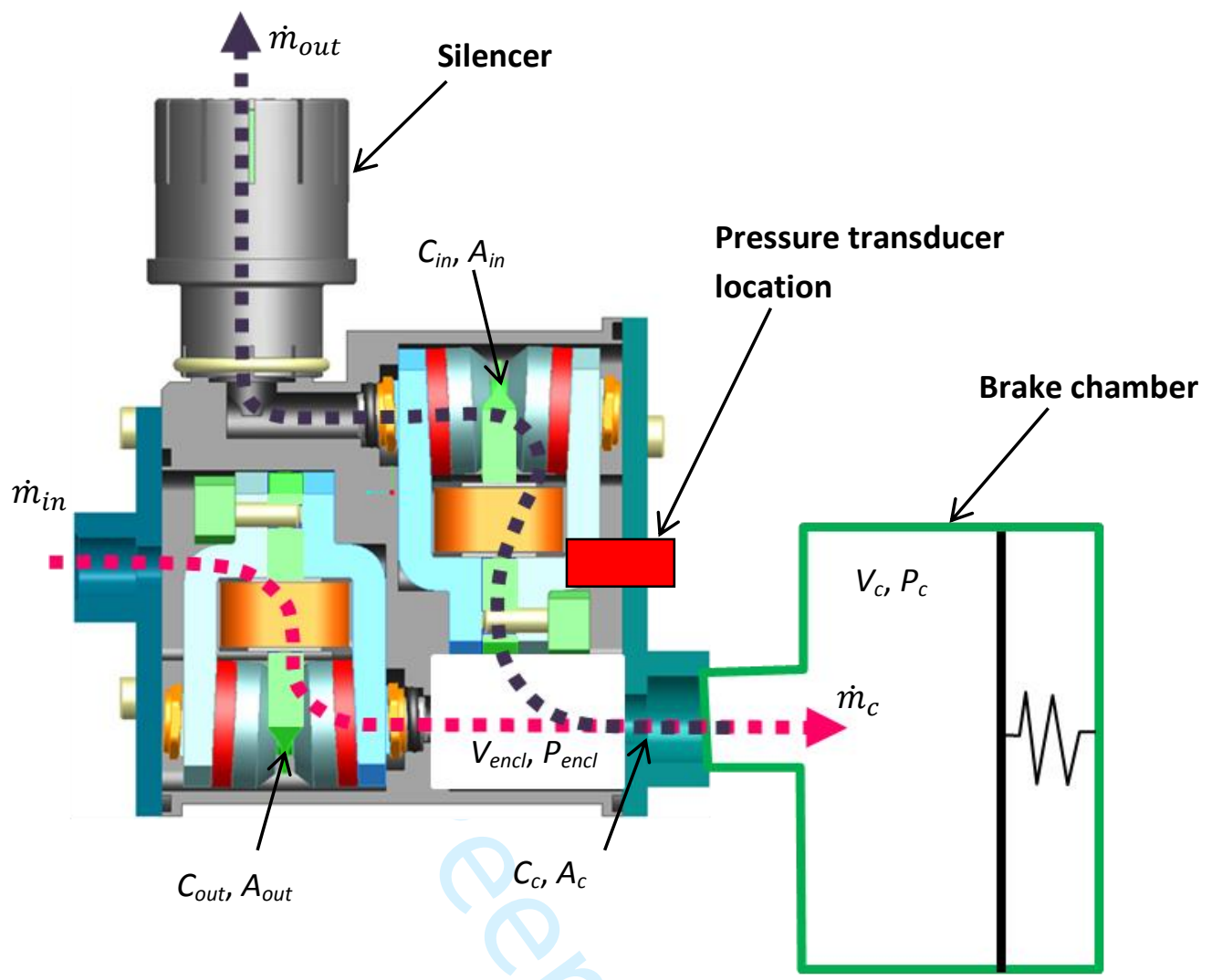

Figure 9: Generation 3 bistable valve enclosure and brake chamber model used in brake chamber pressure observer 


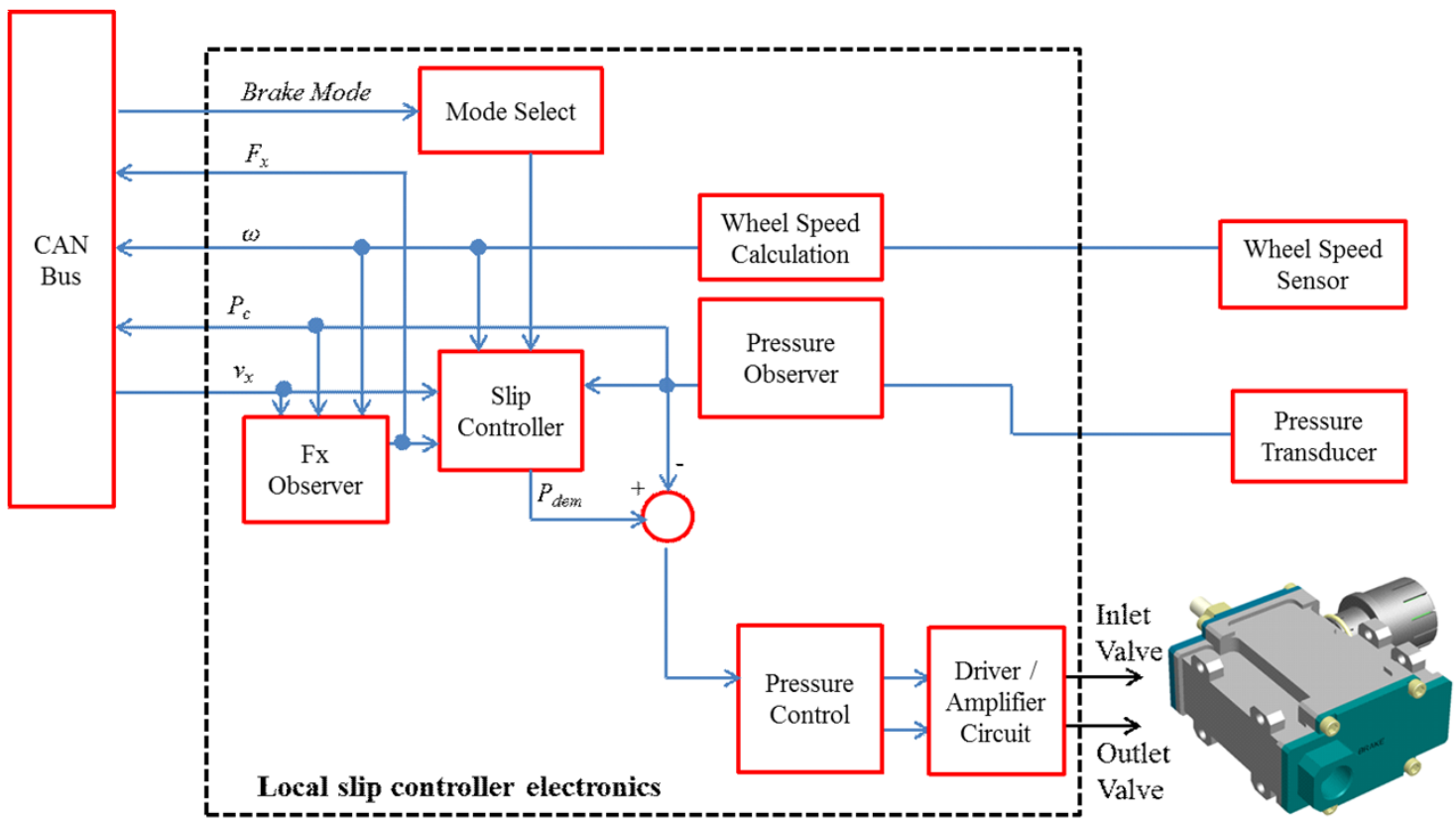

Figure 10: Local wheel controller flow diagram for slip control system with bistable valve hardware 


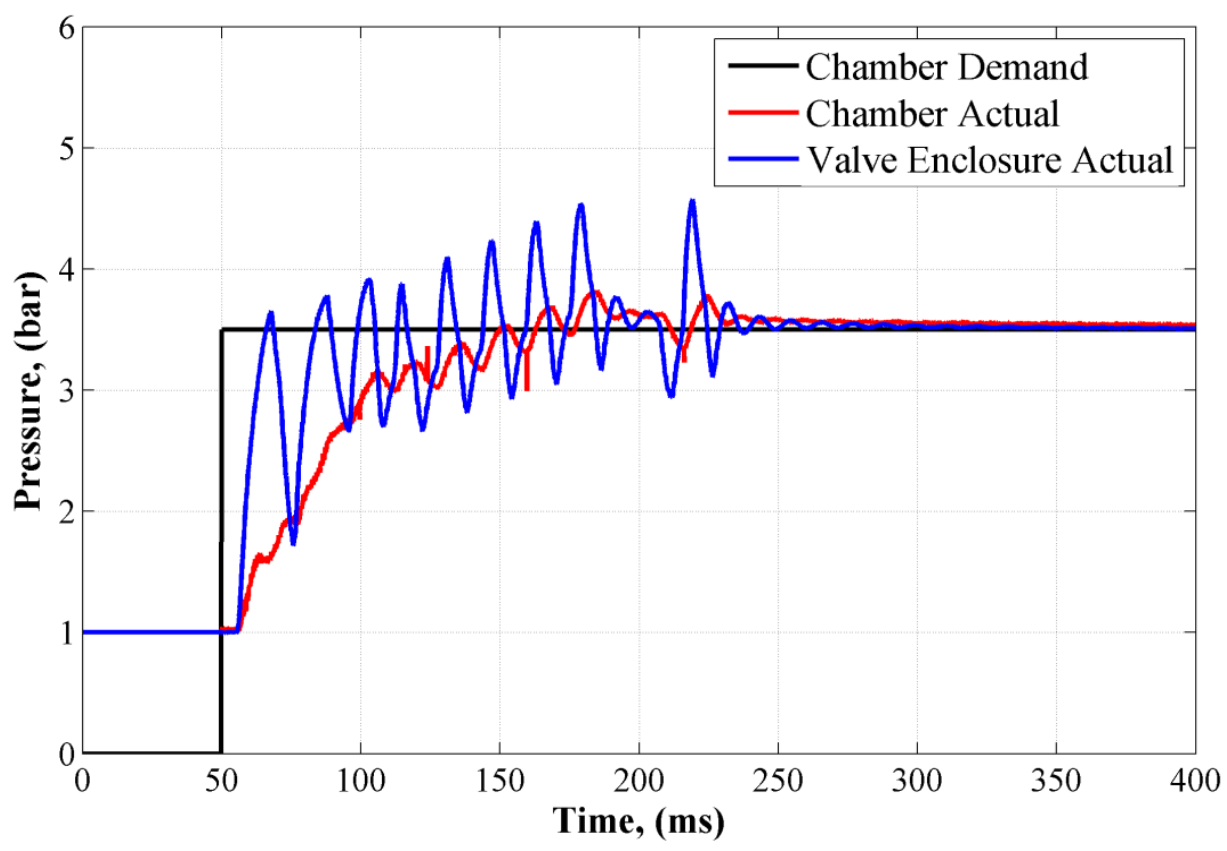

Figure 11: Preliminary pressure controller performance, generation 3 valve enclosure $\left(k_{\text {press }}\right.$ $=1.5 \times 10^{-5} \mathrm{~Pa}^{-1}$ ) 


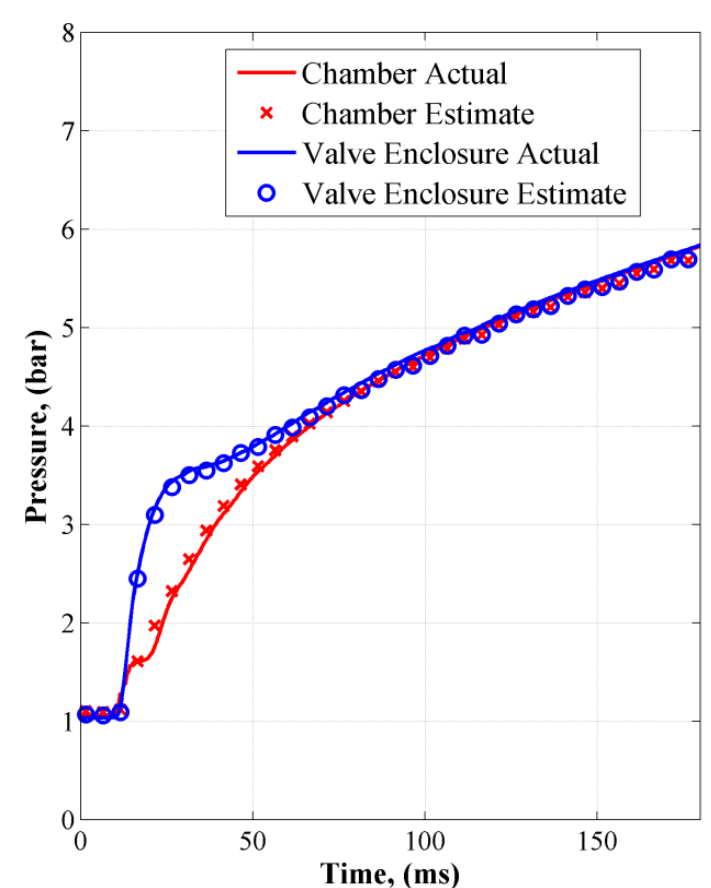

(a)

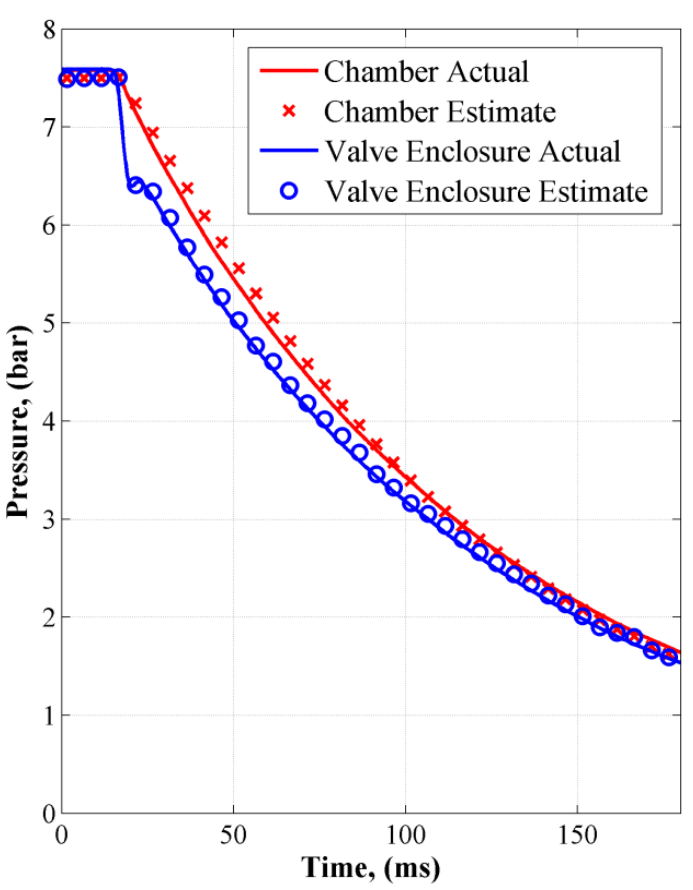

(b)

Figure 12: Brake chamber pressure observer performance: (a) Chamber fill, (b) Chamber exhaust 


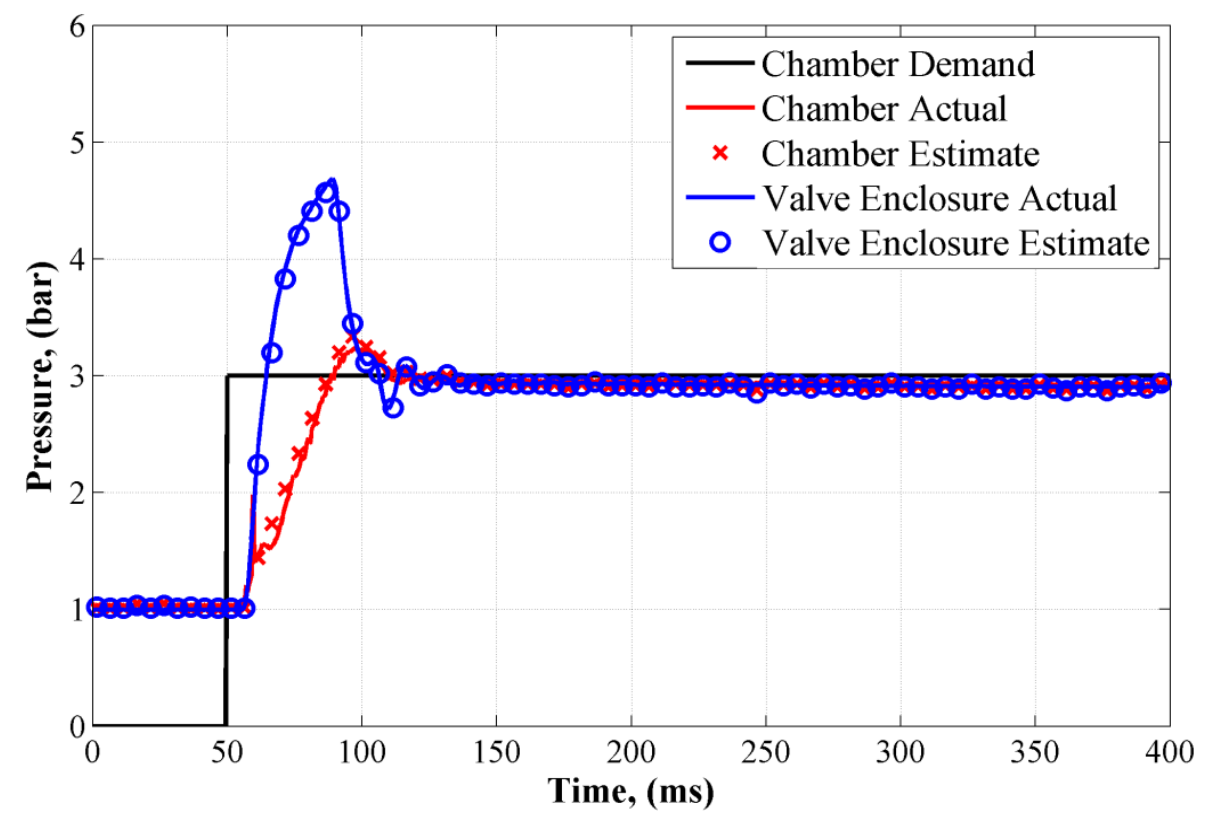

Figure 13: Closed loop pressure control performance of generation 3 valve enclosure, brake chamber pressure observer active $\left(k_{\text {press }}=1.5 \times 10^{-5} \mathrm{~Pa}^{-1}\right)$ 


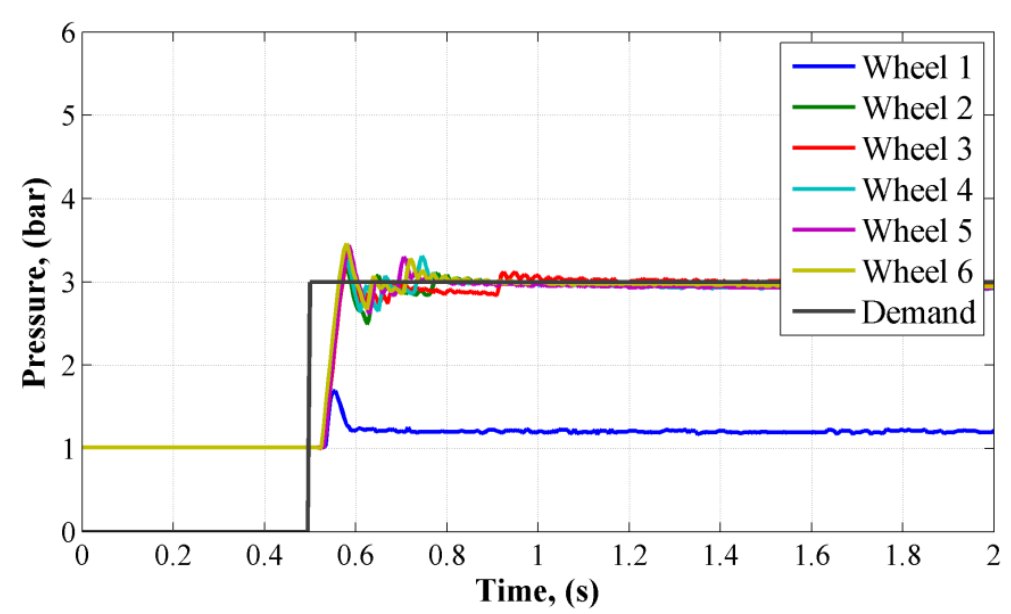

(a)

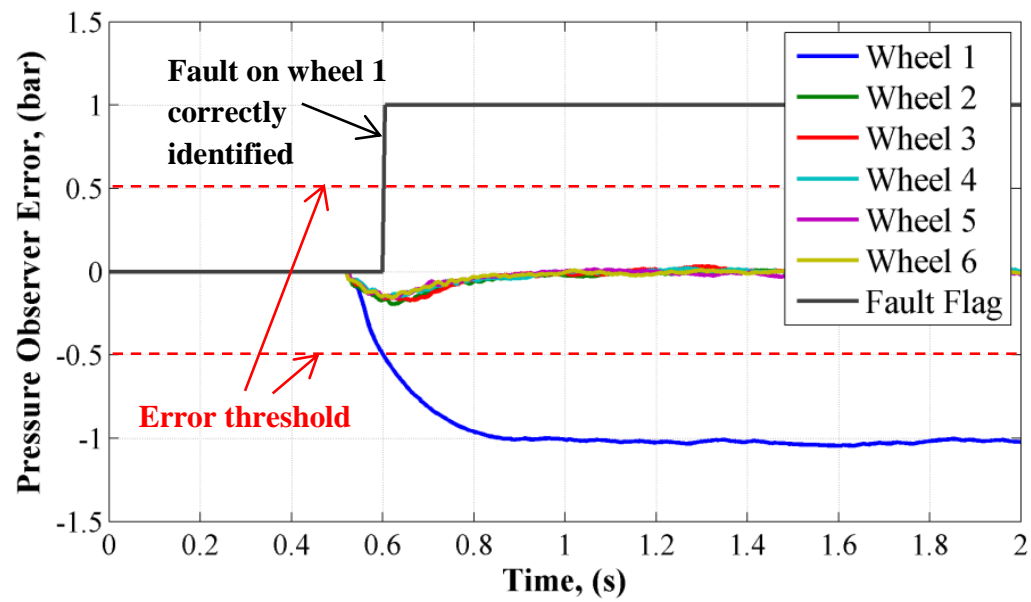

(b)

Figure 14: Fault detection during vehicle pressure control commissioning; (a) Brake chamber pressures, (b) Error measurements generated by brake chamber pressure observer 


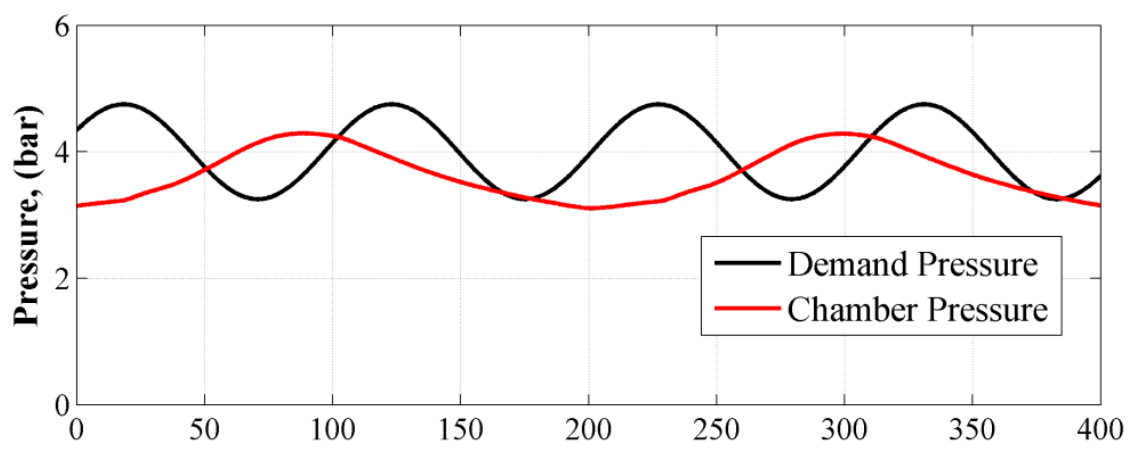

(a)

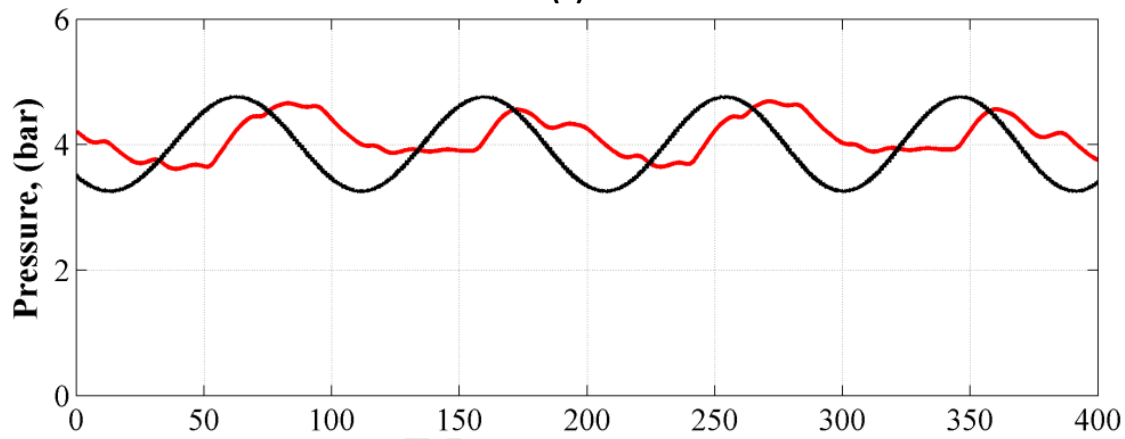

(b)

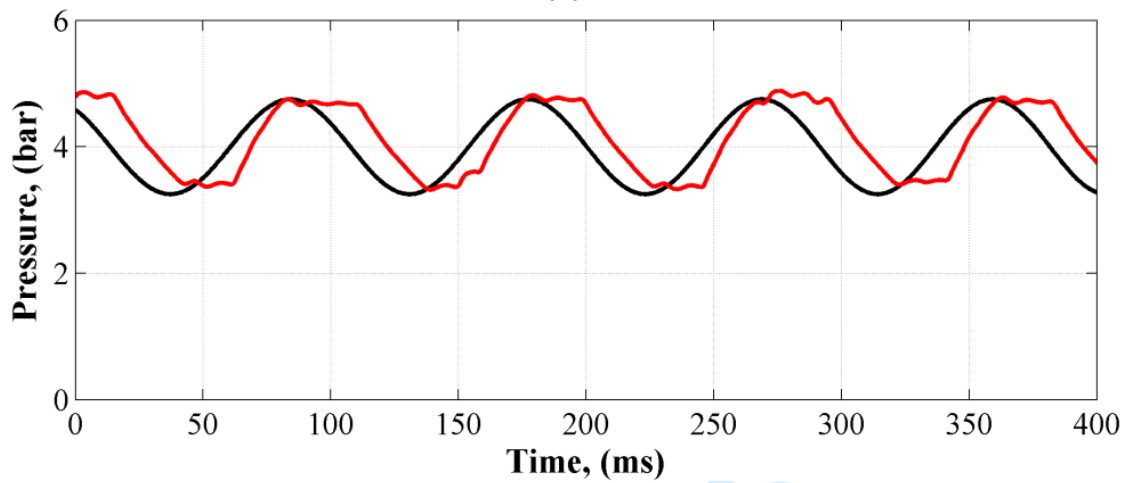

(c)

Figure 15: Pressure controller performance, response to $10 \mathrm{~Hz}$ sinusoidal pressure demand: (a) Conventional EBS hardware, (b) Generation 2 bistable valve system, (c) Generation 3 bistable valve system 

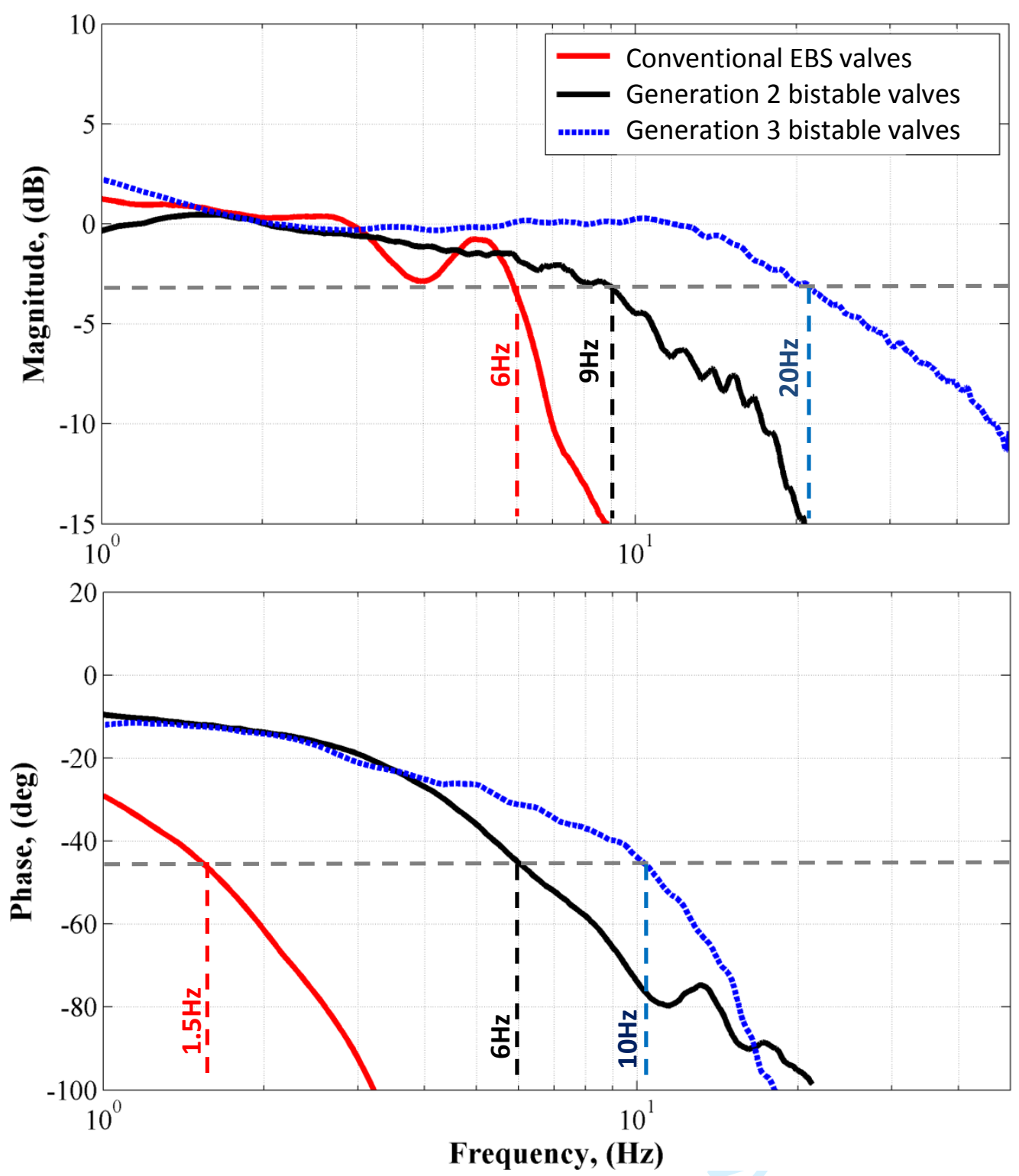

Figure 16: Frequency response obtained from conventional EBS and generation 2 and generation 3 bistable valve systems using HiL test rig (dashed lines indicating bandwidth) 


\section{References}

[1] A. Dunn and R. Hoover, "Class 8 Truck Tractor Braking Performance Improvement Study," U.S. Department of Transportation, 2004.

[2] G. Forkenbrock, M. Flick, and W. Garrot, "A comprehensive light vehicle antilock brake system test track performance evaluation," Society of Automotive Engineers, 1998.

[3] P. Fancher and C. Winkler, "Directional performance issues in evaluation and design of articulated heavy vehicles," Vehicle System Dynamics, vol. 45, pp. 607-647, JulAug 2007.

[4] J. Miller, L. Henderson, and D. Cebon, "Designing and testing an advanced pneumatic braking system for heavy vehicles," Proceedings of the Institution of Mechanical Engineers Part C-Journal of Mechanical Engineering Science, vol. 227, pp. 1715-1729, 2013.

[5] Henderson, L. and Cebon, D. 'Full-scale testing of a novel slip control braking system for heavy vehicles', IMechE, Part D: J. of Auto Eng, Vol. 230(9) 1221-1238, 2016. DOI: http://dx.doi.org/ 10.1177/0954407015604804.

[6] J. I. Miller, T. J. Flack, and D. Cebon, "Modeling the Magnetic Performance of a Fast Pneumatic Brake Actuator," Journal of Dynamic Systems Measurement and ControlTransactions of the ASME, vol. 136, p. 12, Mar 2014.

[7] B. P. Lequesne, "Finite-element analysis of a contat-force solenoid for fluid-flow control," IEEE Transactions on Industry Applications, vol. 24, pp. 574-581, Jul-Aug 1988.

[8] T. Kajima, "Dynamic-model of the plunger type solenoid at deenergizing state," Ieee Transactions on Magnetics, vol. 31, pp. 2315-2333, May 1995.

[9] H. Liu, H. Gu, and D. Chen, "Application of high-speed solenoid valve to the semiactive control of landing gear," Chinese Journal of Aeronautics, vol. 21, pp. 232-240, Jun 2008.

[10] "Cambridge Engineering Selector," ed: Granta Design Ltd, Cambridge, UK, 2013.

[11] D.Cebon, A. Odhams, N. Houghton, W. Wlayslaw, J. Miller, R. Prescott, L. Henderson and L. Potter, 'Electromagnetic Flexure' European Patent 12766136.1 granted 13 March, 2015. US Patent No US 9,404,602 B2 granted Aug 2, 2016.

[12] I. Grant and W. Phillips, Electromagnetism, 2nd ed. Chichester, England: John Wiley \& Sons Ltd, 1995.

[13] P. Campbell, Permanent magnet materials and their application. Cambridge: Cambrdige University Press, 1994.

[14] H. Roters, "Electromagnetic Devices," ed. New York: Chapman \& Hall, 1948.

[15] Structures Databook, 1999.

[16] J. Miller, "Advanced braking systems for heavy vehicles," PhD Dissertation, Cambridge, 2010.

[17] L. Meirovitch, Fundamentals of Vibrations. McGraw-Hill, 2001.

[18] A. Burr, Mechanical Analysis and Design. Amsterdam: Elsevier, 1981.

[19] J. Miller, "Modelling and performance of a pneumatic brake actuator," Proc of the IMechE Part C - J of Mech Eng Sci, Vol 226, No 8, pp2076-2092, August 2012.

[20] J. I. Miller and D. Cebon, "An investigation of the effects of pneumatic actuator design on slip control for heavy vehicles," Vehicle System Dynamics, vol. 51, pp. 139164, Jan 12013.

[21] P. Bigras, K. Khayati, and A. C. C. Acc, "Nonlinear observer for pneumatic system with non negligible connection port restriction," in 20th Annual American Control Conference (ACC), Anchorage, Ak, 2002, pp. 3191-3195. 
[22] "EBS in towing vehicles and buses: System Description," WABCO Vehicle Control Systems, 2011. 